\title{
On some problems of Kobayashi and Lang; algebraic approaches
}

\author{
Claire Voisin
}

\section{Contents}

0. Introduction 54

1. The analytic setting $\quad 59$

1.1. Basic results and definitions $\quad 59$

1.1.1. Definition of the pseudo-metrics/volume forms $\quad 59$

1.1.2. First properties 61

1.1.3. Brody's theorem, applications 63

1.1.4. Relation between volume and metric 67

1.2. Curvature arguments 68

1.2.1. Hyperbolic geometry and the Ahlfors-Schwarz lemma 68

1.2.2. Another definition of the Kobayashi infinitesimal pseudo-metric $\quad 72$

1.2.3. Jet differentials 74

$\begin{array}{ll}\text { 1.3. Conjectures, examples } & 76\end{array}$

1.3.1. Kobayashi's conjecture on volumes $\quad 76$

1.3.2. Conjectures on metrics 80

1.3.3. Campana's construction and conjectures 82

2. Algebraic versions, variants, Lang's conjectures 83

$\begin{array}{ll}\text { 2.1. Subvarieties and hyperbolicity } & 83\end{array}$

2.1.1. Algebraic hyperbolicity 83

2.1.2. Lang's conjectures $\quad 88$

2.1.3. Algebraic measure-hyperbolicity 90

2.2. $K$-correspondences and a variant of Kobayashi's
conjecture

2.2.1. $K$-correspondences $\quad 93$

2.2.2. Existence theorem for $K$-correspondences $\quad 95$ 
2.2.3. A variant of the Kobayashi-Eisenman pseudovolume form

3. General hypersurfaces in projective space

3.1. Hyperbolicity of hypersurfaces and their complements $\mathbf{1 0 1}$

3.1.1. Algebraic hyperbolicity 101

3.1.2. Geometry of the complement 106

3.1.3. Kobayashi's hyperbolicity 108

3.2. Calabi-Yau hypersurfaces 112

3.2.1. Rational curves on Calabi-Yau hypersurfaces 112

3.2.2. Sweeping out a general hypersurface by subvarieties 116

3.2.3. Lang's and Clemens' conjectures contradict 120

References

122

\section{Introduction}

The goal of this survey paper is to present results on hyperbolicity of complex algebraic manifolds, which appeared after the papers [48] and $[42]$, where a number of important and intriguing conjectures were proposed. Since the paper puts the accent on measure hyperbolicity on one hand, and on algebraic methods on the other hands, we hope it will not overlap too much with the beautiful paper [21] by Demailly, except for the basic definitions and starting points.

The basic questions asked in [42], and in a different spirit in [48] concern the relationships between curvature properties of a given complex manifold (or complex algebraic variety) on one hand, and the behaviour of holomorphic maps from disks or polydisks to them, on the other hand, or, from a more algebraic point of view, the (non) existence of subvarieties of certain types. The well-known statements illustrating this are the following :

(1) (Analytic geometry.) A curve of genus $g \geq 2$ admits a metric $\mu$ with Ricci form $i \partial \bar{\partial} \log \mu>0$, and its universal cover is the unit disk. There are no non constant holomorphic maps from $\mathbb{C}$ to it.

(2) (Algebraic geometry.) [47], [7] A Fano variety (i.e a variety with negative canonical bundle, or equivalently, admitting a volume form $\mu$ with $i \partial \bar{\partial} \log \mu<0)$ is rationally connected, that is, through any two points, there passes a rational curve.

The first statement is classical. The second statement has been established only recently in full generality, while it had been known classically for many specific examples. 
In the intermediate range, for example when the canonical bundle is trivial, we meet the very intriguing phenomenon that rational curves cannot cover the variety, as follows from the adjunction formula and the fact that the degree of the normal bundle of a curve which is the generic member of a covering family is non negative, but, at least conjecturally, the variety should be covered by images of non constant holomorphic maps from $\mathbb{C}$.

The problems and partial results presented here are obtained by generalizing and mixing such statements. The first possible generalization of 1 above is of course by looking at higher dimensional manifolds. The study of holomorphic maps from the unit disk $D$ to $X$ led Kobayashi to the definition of the Kobayashi pseudo-distance, later completed by Royden's introduction of the Kobayashi-Royden infinitesimal pseudo-metric. Another possible generalization consists in looking at holomorphic maps from polydisks $D^{n}$ to $X, n=\operatorname{dim} X$, eg at local uniformizations, leading to the Kobayashi-Eisenman pseudo-volume form, which, as shown by Theorem 1.29, might be to some respects easier to deal with than the Kobayashi pseudo-metric.

The Kobayashi-Royden infinitesimal pseudo-metric is 0 on a given tangent vector $u$ at $x \in X$ if and only if there exist holomorphic maps $f: \Delta \rightarrow X$ satisfying $f(0)=x$ and $f_{*}\left(\frac{\partial}{\partial t}\right)$ is arbitrarily large, proportional to $u$. Similarly the Kobayashi-Eisenman pseudo-volume form is 0 at a point $x \in X$ if there exist holomorphic maps $f: D^{n} \rightarrow X, n=$ $\operatorname{dim} X, f(0)=x$, with arbitrarily large Jacobian determinant at 0 .

A basic result due to Brody, ( Theorem 1.15), is the fact that for compact $X$, the Kobayashi infinitesimal pseudo-metric is degenerate at some point $x \in X$ if and only if there exists a non constant holomorphic map $f: \mathbb{C} \rightarrow X$. Here "degenerate at $x$ " means that there exists a non zero tangent vector $u \in T_{X, x}$, and a sequence of holomorphic maps

$$
f_{k}: D \rightarrow X
$$

with $\lim _{k \rightarrow \infty} f_{k}(0)=x$ and $f_{k *}\left(\frac{\partial}{\partial t}\right)=\mu_{k} u_{k}$, with

$$
\lim _{k \rightarrow \infty} \mu_{k}=\infty, \lim _{k \rightarrow \infty} u_{k}=u .
$$

The main conjectures which emerge from [48], [42], [31], are the following (we refer to the text for the definitions needed):

CONJECTURE 0.1. (Kobayashi) Let $X$ be a projective variety which is not of general type. Then its pseudo-volume forms $\Psi_{X}$ and/or $\widetilde{\Psi}_{X}$ vanish almost everywhere.

This conjecture is a kind of converse to Griffiths-Kobayashi-Ochiai Theorem 1.29, which says that the Kobayashi-Eisenman pseudo-volume 
form $\Psi_{X}$ or its Yau variant $\widetilde{\Psi}_{X}$ is non zero on a Zariki dense open set of $X$, if $X$ is of general type.

ConjeCture 0.2. (Lang) Let $X$ be a projective variety which is not of general type. Then $X$ is swept out by images of non-constant rational maps from an abelian variety to $X$.

This conjecture is an attempt to understand algebraically Kobayashi's conjecture above. Indeed, if $X$ is rationally swept out by abelian varieties, then $\widetilde{\Psi}_{X}=0$ almost everywhere (cf Lemma 1.42). However, Corollary 3.34 shows that this conjecture, for general Calabi-Yau hypersurfaces in projective space, is in contradiction with Clemens' conjecture on rational curves in such varieties, which is based on a deformation argument.

ConjeCture 0.3. (Lang) Let $X$ be any complex algebraic variety. Then if $X$ is not Kobayashi hyperbolic, there exists a non-constant rational map from an abelian variety to $X$.

In fact, Lang conjectured more precisely that the locus swept out by entire curves is equal to the locus swept out by images of non constant rational maps from an abelian variety to $X$, and that this locus is equal to $X$ if and only if $X$ is not of general type. Note that this conjecture is not true if $X$ is not projective $[\mathbf{9}]$.

Conjecture 0.4. (Green-Griffiths) Let $X$ be a projective complex manifold which is of general type. Then an entire curve is not Zariski dense in $X$.

A more optimistic version asks whether there exists a proper algebraic subset containing all entire curves. This conjecture is supported by the case of subvarieties of complex tori, (Bloch's Theorem, see also Green and Griffiths, [31]) and by the case of surfaces, where it is proved by McQuillan for surfaces of general type satisfying $c_{1}^{2}>c_{2}$ (cf [50] and Theorem 2.7). Furthermore, for surfaces of general type, without any conditions on the Chern numbers, Green and Griffiths show that there is an algebraic differential equation satisfied by all the holomorphic maps from $\mathbb{C}$ to $S$.

ConjeCture 0.5. (Kobayashi) Let $X$ be a projective variety with trivial canonical bundle. Then its Kobayashi pseudo-distance vanishes.

This is largely open. This is known for surfaces, using the classification and the fact that $K 3$ surfaces are swept out by elliptic curves. There is a more general conjecture by Campana characterizing projective varieties for which the Kobayashi pseudo-distance vanishes (cf section 1.3.3). 
ConjeCture 0.6. (Demailly) A complex projective variety is Kobayashi hyperbolic if and only if it is algebraically Kobayashi hyperbolic.

Here the notion of algebraic Kobayashi hyperbolicity is as follows : $X$ is said to be algebraically hyperbolic if the geometric genus of curves in $X$ grows linearly with their degree with respect to a (any) ample line bundle on $X$. It is immediate to see that Kobayashi hyperbolicity implies algebraic Kobayashi hyperbolicity (cf Proposition 2.1). Algebraic Kobayashi hyperbolicity is much easier to prove (see section 3.1.1), so this conjecture is presumably very hard. One might also ask whether it can combined with Conjecture 0.4 : in a variety of general type, does there exist a closed algebraic subset containing all curves $C$ satisfying $g(C) / \operatorname{deg} C \leq A$ for $A$ small enough?

ConjeCture 0.7. (Kobayashi) Let $X$ be a general hypersurface of large degree $d$ in $\mathbb{P}^{n+1}$. Then $X$ is Kobayashi hyperbolic.

This conjecture has been solved recently by Demailly-Elgoul and independently McQuillan for surfaces, and then by Siu in the general case. A more precise version of it asks whether the hypersurfaces as above are Kobayashi hyperbolic when $d \geq 2 n+1, n \geq 2$. A related problem is the (algebraic) study of Green-Griffiths conjecture for general hypersurfaces in the range $n+3 \leq d \leq 2 n-1$ in $\mathbb{P}^{n+1}$. Indeed, in this range we know that the hypersurfaces are of general type but contain lines, so are not Kobayashi hyperbolic. One important and presumably accessible question is : is the degree of rational curves in general hypersurfaces in this range bounded? The answer is known when the degree $d$ satisfies the condition $d \geq \frac{3 n+2}{2}$, by Pacienza's Theorem [57], later on generalized by Clemens and Ran [16] : the only rational curves existing in general hypersurfaces in $\mathbb{P}^{n+1}$ in the range $\frac{3 n+2}{2} \leq d \leq 2 n-1$ are lines.

The related progresses presented here are the following ;

(1) (Demailly-Lempert-Shiffmann, see section 1.2.2) The Kobayashi infinitesimal pseudo-metric of a projective algebraic variety can be computed using algebraic curves in $X$, together with their Poincaré metric.

(2) (Voisin, see section 2.2) Replacing holomorphic maps by $K$ correspondences (cf section 2.2.1), we get a variant of the Kobayashi-Eisenman pseudo-volume form (cf section 2.2.3). On one hand it satisfies the analogue Griffiths, Kobayashi-Ochiai theorem 1.29, that is, it is non-degenerate on a Zariski open set for a variety of general type. On the other hand it vanishes for many varieties which are not of general type. 
(3) (Voisin, see section 3.2.3) If $X$ is a general Calabi-Yau hypersurface in projective space, then $X$ is not swept out by images of rational maps from abelian varieties of dimension $\geq 2$ to $X$. As a consequence, Lang's conjecture 0.2 and Clemens conjecture on the finiteness of rational curves of given degree in a general quintic threefold, contradict.

(4) (Clemens, Ein, Voisin, see section 3.1.1) Let $X \subset \mathbb{P}^{n+1}$ be a general hypersurface of degree $d \geq 2 n+1$. Then $X$ is algebraically Kobayashi hyperbolic.

(5) (Demailly-El Goul, McQuillan for degree $\geq 36$, see section 3.1.3) A general surface in $\mathbb{P}^{3}$ of degree $\geq 21$ is Kobayashi hyperbolic.

(6) (Siu, see section 3.1.3)A generic hypersurface of sufficiently high degree in projective space is Kobayashi hyperbolic.

As one can see, many problems from the above list remain open. The most important are maybe conjectures 0.4 and 0.1 , which together provide a very nice picture of the whole subject.

From a purely algebraic point of view, the most intriguing questions which have been mentioned here in the case of hypersurfaces, are related to Bogomolov's Theorem [4], saying that on a surface of general type satisfying the inequality

$$
c_{1}^{2}>c_{2}
$$

there are only finitely many families of curves of any given genus. This theorem is obviously related to Conjecture 0.4 , and should be true without the assumptions on the Chern classes. However it is still unknown if a smooth quintic surface in $\mathbb{P}^{3}$ can have infinitely many rational curves (see more generally Conjecture 3.9).

A part from these results and the sketch of their proofs, the survey will present a number of examples illustrating the conjectures. We shall also provide the details of a few basic points, like Ahlfors-Schwarz Lemma or Brody's reparametrization Lemma, and their main classical consequences, like the weak Bloch Theorem or the Griffiths-KobayashiOchiai Theorem, hoping to make the paper as useful an instructive as possible.

There has been recent important developments [29], [30] of Lang's ideas, which we have completely omitted here, concerning the number theoretic vision of these problems. Namely relate the analytic properties of a complex variety, to the behavior of its rational points over non algebraically closed fields.

One reason not to expose these results in this paper is the fact that they concern rationally connected varieties, and more precisely, 
working over function fields, families of them, while the dichotomy considered here is being of general type or not.

Thanks. I would like to thank Harvard University and MIT for inviting me to deliver lectures at the seminar Current developments in Mathematics 2003, which led me to write-up these notes.

\section{The analytic setting}

1.1. Basic results and definitions. We introduce in this preliminary section the basic definitions of the Kobayashi-Eisenman Finsler pseudo-metrics on the spaces of holomorphic $k$-vectors and state a few properties. The remainder of the text will be mainly devoted to the two cases of the volume $(k=n)$ and the Kobayashi-Royden infinitesimal pseudo-metric $(k=1)$, with emphasis on the volume case in chapter 2. We also explain Brody's reparametrization theorem and conclude with a simple statement concerning the relation between volumes and metrics.

1.1.1. Definition of the pseudo-metrics/volume forms. Let $X$ be a complex manifold. Kobayashi [41] introduces a pseudo-distance $d_{K, X}$ on $X$, whose infinitesimal version is a Finsler pseudo-metric $d_{K, X}^{\text {inf }}$ defined by Royden on the tangent space $T_{X}$, and a pseudo-volume form $\Psi_{X}$, i.e, a pseudo-Hermitian norm on $\bigwedge^{n} T_{X}, n=\operatorname{dim} X$. These objects have been generalized by Eisenman $[\mathbf{2 7}]$ to give $p$-pseudo-volume forms defined on holomorphic decomposable $p$-vectors of $X$.

Definition 1.1. (1) The Kobayashi-Royden infinitesimal pseudometric $d_{K, X}^{\text {inf }}$ is defined by

$$
d_{K, X}^{i n f}(u)=\frac{1}{\lambda}, \lambda=\sup _{\phi}\left\{|\mu|, \phi_{*}\left(\frac{\partial}{\partial t}\right)=\mu u\right\},
$$

for $u \in T_{X, x}$. Here $\phi$ runs over the set of holomorphic maps from the unit disk $D$ to $X$, such that $\phi(0)=x$.

(2) The Kobayashi pseudo-volume form $\Psi_{X}$ is the pseudo-volume form whose associated Hermitian pseudo-norm on $\bigwedge^{n} T_{X}$ is defined by

$\Psi_{X}(\zeta)=\frac{1}{\lambda}, \lambda=\sup _{\phi}\left\{|\mu|, \phi_{*}\left(\frac{\partial}{\partial t_{1}} \wedge \ldots \wedge \frac{\partial}{\partial t_{1}}\right)=\mu \zeta\right\}$,

for $\zeta \in \bigwedge^{n} T_{X, x}, n=\operatorname{dim} X$. Here $\phi$ runs over the set of holomorphic maps from $D^{n}$ to $X$ such that $\phi(0)=x$.

(3) More generally the Eisenman p-pseudo-volume form $\Psi_{X}^{p}$ is defined as in 2, replacing $n=\operatorname{dim} X$ by any $p$ comprised between 
1 and $n$. It is then defined only on p-vectors $\zeta \in \bigwedge^{p} T_{X, x}$ which are decomposable, that is $\zeta=u_{1} \wedge \ldots \wedge u_{p}$.

We clearly have

$$
\Psi_{X}^{1}=d_{K, X}^{i n f}, \Psi_{X}^{n}=\Psi_{X}
$$

EXAMPlE 1.2. If $X=\mathbb{C}^{n}$, we have $\Psi_{X}^{p}=0, \forall p \geq 1$. Indeed, there is a polydisc $D^{p} \subset \mathbb{C}^{n}$ centered at any possible point and with arbitrary given tangent space. Using then affine maps $v \mapsto \lambda v+v_{0}$ with adequate $v_{0}$, and taking $\lambda$ arbitrarily large, provides such polydisk with arbitrarily large associated $p$-vector at 0 , centered at any given point and having arbitrary given tangent space at this point.

In the sequel we shall be mainly interested in the two extremal cases $p=1, p=n$.

It is possible to introduce integrated versions of the above notions.

Definition 1.3. The Kobayashi measure is the measure defined by the pseudo-volume form $\Psi_{X}$. It is well defined since $\Psi_{X}$ can be defined locally as a dominated supremum of differentiable volume forms.

In order to define the Kobayashi pseudo-distance, let us recall the Poincaré metrics on the disks :

Definition 1.4. Let $D_{r}$ be the disk of radius $r$ in $\mathbb{C}$. Then the Poincaré metric \|\|$_{P_{r}}$ on $D_{r}$ is defined by its Kähler form :

$$
\Omega_{r}=\frac{i}{2} \frac{r^{4}}{\left(r^{2}-|z|^{2}\right)^{2}} d z \wedge d \bar{z} .
$$

Note that \|\|$_{P_{r}}$ is equal to the Euclidean metric at 0. Furthermore, when $r$ tends to infinity, it converges uniformly on compact sets $K \subset \mathbb{C}$ to the Euclidean metric.

For $r=1$, we shall denote by \|\|$_{P}$ the Poincaré metric and $d_{P}$ the Poincaré distance on the unit disk.

Definition 1.5. The Kobayashi pseudo-distance $d_{K, X}$ is defined by

$$
d_{K, X}(x, y)=\inf _{\phi_{i}, p_{i}, q_{i}} \sum_{i} d_{P}\left(p_{i}, q_{i}\right),
$$

where the $\phi_{i}$ 's, $i=1, \ldots, N$, are $N$ holomorphic maps from $D$ to $X$, and the points $p_{i}, q_{i} \in D$ satisfy :

$$
\phi_{1}\left(p_{1}\right)=x, \phi_{N}\left(q_{N}\right)=y, \phi_{i}\left(q_{i}\right)=\phi_{i+1}\left(p_{i+1}\right), i<N .
$$

Royden [58] proved that $d_{K, X}$ is the distance deduced from $d_{K, X}^{i n f}$ by integration over pathes : 
Theorem 1.6. (Royden) For $\gamma:[0,1] \rightarrow X$ a differentiable path in $X$, the length $\int_{[0,1]} d_{K, X}^{\text {inf }}(\dot{\gamma}) d t$ is well defined, and one has

$$
d_{K, X}(x, y)=\inf _{\gamma, \gamma(0)=x, \gamma(1)=y} \int_{[0,1]} d_{K, X}^{i n f}(\dot{\gamma}) d t .
$$

REMARK 1.7. A simple but important point in the proof of the theorem above, is the fact that for $X=D$, the Kobayashi pseudometric is equal to the Poincaré metric \|\|$_{P}$, a fact which will be proved in 1.2.1.

Definition 1.8. $X$ is said to be Kobayashi hyperbolic if $d_{K, X}$ is actually a distance on $X$. As we shall see later on, if $X$ is compact, it is equivalent to the fact that $d_{K, X}^{i n f}$ is equivalent to any Hermitian norm on $T_{X}$.

Definition 1.9. $X$ is said to be measure hyperbolic if the Kobayashi measure does not vanish on any open set, or equivalently $\Psi_{X}$ is non zero almost everywhere.

1.1.2. First properties. The first obvious property satisfied by the pseudo-metrics or distances defined in the previous section is the decreasing property under holomorphic maps.

Lemma 1.10. Let $\phi: X \rightarrow Y$ be a holomorphic map. Then for any integer $p$, we have

$$
\phi^{*}\left(\Psi_{Y}^{p}\right) \leq \Psi_{X}^{p}
$$

This makes sense, using the maps $\wedge^{p} \phi_{*}$, which send decomposable $p$-vectors of $X$ at $x$ to decomposable $p$-vectors of $Y$ at $\phi(x)$.

Let us now consider the behavior of $d_{K}$ and $\Psi$ under products.

Proposition 1.11. We have

$$
d_{K, X \times Y}=\sup \left\{p r_{1}^{*} d_{K, X}, p r_{2}^{*} d_{K, Y}\right\} .
$$

Proof. Let $x_{1}, x_{2} \in X, y_{1}, y_{2} \in Y$. Assume that $d_{K, X}\left(x_{1}, x_{2}\right) \leq$ $d_{K, Y}\left(y_{1}, y_{2}\right)$. For any $\epsilon>0$ there exists $\phi_{i}: D \rightarrow X, p_{i}, q_{i} \in D, i=$ $1, \ldots, N$ and $\psi_{i}: D \rightarrow X, p_{i}^{\prime}, q_{i}^{\prime} \in D, i=1, \ldots, N$ such that

$$
\begin{aligned}
& \phi_{1}\left(p_{1}\right)=x_{1}, \phi_{N}\left(q_{N}\right)=x_{2}, \phi_{i}\left(q_{i}\right)=\phi_{i+1}\left(p_{i+1}\right), i<N, \\
& \psi_{1}\left(p_{1}^{\prime}\right)=y_{1}, \psi_{N}\left(q_{N}^{\prime}\right)=y_{2}, \psi_{i}\left(q_{i}^{\prime}\right)=\psi_{i+1}\left(p_{i+1}^{\prime}\right), i<N,
\end{aligned}
$$

and satisfying

$$
\begin{gathered}
d_{P}\left(p_{i}, q_{i}\right) \leq d_{P}\left(p_{i}^{\prime}, q_{i}^{\prime}\right) \\
\sum_{i} d_{P}\left(p_{i}, q_{i}\right) \leq d_{K, X}\left(x_{1}, x_{2}\right)+\epsilon, \sum_{i} d_{P}\left(p_{i}^{\prime}, q_{i}^{\prime}\right) \leq d_{K, Y}\left(y_{1}, y_{2}\right)+\epsilon .
\end{gathered}
$$


Now we observe that since $d_{P}\left(p_{i}, q_{i}\right) \leq d_{P}\left(p_{i}^{\prime}, q_{i}^{\prime}\right)$, there exists a holomorphic map $\chi_{i}: D \rightarrow D \times D$ sending $p_{i}^{\prime}, q_{i}^{\prime}$ to $\left(p_{i}, p_{i}^{\prime}\right),\left(q_{i}, q_{i}^{\prime}\right)$ respectively. But then it follows from the definition of $d_{K, X \times Y}$ that

$$
d_{K, X \times Y}\left(\left(x_{1}, y_{1}\right),\left(x_{2}, y_{2}\right)\right) \leq \sum_{i} d_{P}\left(p_{i}^{\prime}, q_{i}^{\prime}\right) \leq d_{K, Y}\left(y_{1}, y_{2}\right)+\epsilon .
$$

Letting $\epsilon$ tend to 0 , we have proved that

$$
d_{K, X \times Y}\left(\left(x_{1}, y_{1}\right),\left(x_{2}, y_{2}\right)\right) \leq d_{K, Y}\left(y_{1}, y_{2}\right) .
$$

The reverse inequality follows from Lemma 1.10.

As for volume forms, we have the following :

Proposition 1.12. We have

$$
\begin{gathered}
\Psi_{X \times Y} \leq p r_{1}^{*} \Psi_{X} \otimes p r_{2}^{*} \Psi_{Y}, \\
\Psi_{X \times Y} \geq C p r_{1}^{*} \Psi_{X} \otimes p r_{2}^{*} \Psi_{Y},
\end{gathered}
$$

for a constant $C$ which depends only on the dimensions of $X$ and $Y$.

Proof. The first inequality is obvious, since a holomorphic map $\phi_{1}: D^{p} \rightarrow X, p=\operatorname{dim} X$ and a holomorphic map $\phi_{2}: D^{q} \rightarrow Y, q=$ $\operatorname{dim} Y$ provide a holomorphic map $\phi=\left(\phi_{1}, \phi_{2}\right): D^{p+q} \rightarrow X \times Y, p+$ $q=\operatorname{dim} X \times Y$, whose Jacobian determinant in product holomorphic coordinates is the tensor product of the Jacobian determinants of $\phi_{1}$ and $\phi_{2}$.

As for the reverse inequality, let $\phi: D^{n} \rightarrow X \times Y, n=p+q$ be a holomorphic map. For each partition

$$
\{1, \ldots, n\}=I \sqcup J,
$$

we get two maps

$$
\phi_{I}: D^{p} \rightarrow X, \phi_{J}: D^{q} \rightarrow Y
$$

where $\phi_{I}$ is obtained by restricting $\phi$ to $0 \times \ldots \times D \times \ldots$, where the $D$ 's are put in the $i$-th positions, and then projecting to $X$, and similarly for $\phi_{J}$.

Now, in product holomorphic coordinates, the Jacobian matrix $J_{\phi_{I}}$ of $\phi_{I}$ is the matrix whose rows are the first $p$-lines of the one of $\phi$, and whose columns are the columns of indices $i \in I$. The formula

$$
\operatorname{det} J_{\phi}=\sum_{I, J} \epsilon_{I, J} \operatorname{det} J_{\phi_{I}} \operatorname{det} J_{\phi_{J}}
$$

for adequate signs $\epsilon_{I, J}$ shows that

$$
\left|\operatorname{det} J_{\phi}\right| \leq \sum_{I, J}\left|\operatorname{det} J_{\phi_{I}} \operatorname{det} J_{\phi_{J}}\right|
$$




$$
\leq C_{n}^{p} \sup _{I, J}\left|\operatorname{det} J_{\phi_{I}}\right| \cdot\left|\operatorname{det} J_{\phi_{J}}\right|
$$

Hence, for some partition $(I, J)$, we have

$$
C^{-1}\left|\operatorname{det} J_{\phi_{I}}\right| \cdot\left|\operatorname{det} J_{\phi_{J}}\right| \geq \operatorname{det} J_{\phi},
$$

where $C=\frac{1}{C_{n}^{p}}$. It follows that

$$
\Psi_{X \times Y} \geq C p r_{1}^{*} \Psi_{X} \otimes p r_{2}^{*} \Psi_{Y}
$$

\section{Yau's variant.}

In [74], Yau introduces an important variant of the $\Psi_{X}^{p}$ 's that we will denote by $\widetilde{\Psi}_{X}^{p}$.

Definition 1.13. The p-pseudo-volume form $\widetilde{\Psi}_{X}^{p}$ is the semi-positive real function defined on decomposable p-vectors $u \in \bigwedge^{p} T_{X, x}$ by the formula

$$
\widetilde{\Psi}_{X}^{p}(u)=\frac{1}{\lambda}, \lambda=\sup _{\phi}\left\{|\mu|, \phi_{*}\left(\frac{\partial}{\partial t_{1}} \wedge \ldots \wedge \frac{\partial}{\partial t_{1}}\right)=\mu \zeta\right\} .
$$

Here $\phi$ runs over the set of meromorphic maps from $D^{p}$ to $X$, defined near 0 , and such that $\phi(0)=x$.

Clearly, for $p=1$ and $X$ compact, Yau's and Kobayashi's pseudometrics coincide. In general it is not clear at all whether they can be compared, except for the obvious inequality $\widetilde{\Psi}_{X}^{p} \leq \Psi_{X}^{p}$. It is obvious from the definition that the $\widetilde{\Psi}$ satisfy the decreasing property with respect to meromorphic maps:

Lemma 1.14. Let $\phi: X \rightarrow Y$ be a meromorphic map. Then for any $p \leq \operatorname{dim} Y$ we have

$$
\phi^{*}\left(\Psi_{Y}^{p}\right) \leq \Psi_{X}^{p}
$$

away from the indeterminacy locus of $\phi$.

1.1.3. Brody's theorem, applications. If there exists a non constant holomorphic map $f: \mathbb{C} \rightarrow X$, the Kobayashi infinitesimal pseudometric $d_{K, X}^{\text {inf }}$ vanishes on the tangent vectors to $\operatorname{Im} f$, because of the decreasing property and the fact the $d_{K}^{\text {inf }}$ is 0 for $\mathbb{C}$. Hence it follows that $X$ is not Kobayashi hyperbolic. Brody's theorem [5] provides a converse to this :

THEOREM 1.15. (Brody) If $X$ is compact and the Kobayashi infinitesimal pseudometric is degenerate, then there exists a non constant holomorphic map $f: \mathbb{C} \rightarrow X$. 
Here "degenerate" means that there does not exist a constant $C$ (depending of a given Hermitian metric $h$ on $X$ ), such that

$$
d_{K, X}^{\text {inf }}(u) \geq C|u|_{h}, \forall u \in T_{X} .
$$

Proof. If $d_{K, X}^{\text {inf }}$ is degenerate, there exists a sequence of holomorphic maps $\phi_{n}: D_{r_{n}} \rightarrow X$, satisfying

$$
\left|d \phi_{n}(0)\left(\frac{\partial}{\partial t}\right)\right|_{h}=1, \lim _{n \rightarrow \infty} r_{n}=\infty .
$$

The proof of the theorem uses then the following lemma :

LEMMA 1.16. (Brody's reparametrization Lemma) Under the above assumption, there exists a sequence of holomorphic maps $\psi_{n}: D_{r_{n}} \rightarrow$ $X$, satisfying the properties :

$$
\left|d \psi_{n}(0)\left(\frac{\partial}{\partial t}\right)\right|_{h}=1=\operatorname{Sup}_{z \in D_{r_{n}}}\left\|d \phi_{n}(z)\right\|_{P_{r_{n}}, h} .
$$

Here the norm \|\|$_{P_{r_{n}, h}}$ on Hom $\left(T_{D_{r_{n}}, z}, T_{X, \psi_{n}(z)}\right)$ is induced by $h$ and by the Poincaré metric \|\|$_{P_{r_{n}}}$ (see Definition 1.4).

Admitting the Lemma, we conclude as follows : the sequence of maps $\psi_{n}$, which is defined on any compact set $K \subset \mathbb{C}$ for $n$ large enough depending on $K$, is equicontinuous by the second bound in (1.2), and because the Poincaré metrics \|\|$_{P_{r_{n}}}$ converge uniformly on compact sets to the Euclidean metric. Hence we can extract a subsequence which converges uniformly on compact sets to a holomorphic map

$$
f: \mathbb{C} \rightarrow X,
$$

which is non constant, because of the first equality in (1.2).

Remark 1.17. Since the Poincaré metric on $D_{r_{n}}$, which is defined on $D_{R}$ for $n$ large, converges uniformly on compact sets to the Euclidean metric on $\mathbb{C}$, the limiting map $f$ satisfies the property that

$$
\operatorname{Sup}_{z}\|d f(z)\|_{E, h}=1,
$$

where the norm \|\|$_{E, h}$ on $\operatorname{Hom}\left(T_{\mathbb{C}, z}, T_{X, f(z)}\right)$ is induced by $h$ and by the Euclidean metric on $\mathbb{C}$.

Proof of the Lemma. For each $t \in[0,1]$, define

$$
s(t)=\operatorname{Sup}_{z \in D_{r_{n}}}\left\|(\phi \circ t)_{*}\right\|_{P_{r_{n}}, h},
$$

where the norm here is computed w.r.t the metric $h$ on $X$ and the Poincaré metric \|\|$_{P_{r_{n}}}$ on $D_{r_{n}}$. We use here $t$ to denote the multiplication by $t$ acting on $D_{r_{n}}$. One shows easily that $s$ is a continuous 
function of $t$. On the other hand, we have $s(0)=0$ and $s(t) \geq 1$, since the Poincaré metric is equal to the Euclidean metric at 0 , and by assumption (1.1). Hence for some $t_{n} \in(0,1]$, we have $s\left(t_{n}\right)=1$. The map $\psi_{n}$ will then be defined as follows : if $t_{n}=1$ one puts $\psi_{n}=\phi_{n}$. If $t_{n}<1$, it is clear that the supremum which defines $s\left(t_{n}\right)$ is realized at some point $y \in D_{r_{n}}$ because in this case the function $\left\|(\phi \circ t)_{*}\right\|_{P_{r_{n}}, h}$ vanishes on the boundary of $D_{r_{n}}$. Then one puts

$$
\psi_{n}=\phi_{n} \circ t_{n} \circ g
$$

where $g$ is an automorphism of the disk $D_{r_{n}}$ sending 0 to $y$. Since Aut $D$ acts preserving the Poincaré metrics, $\psi_{n}$ satisfies the assumptions (1.2).

A simple application of this theorem, which is given in [33], is the following weak version of Bloch's theorem :

TheOREM 1.18. Let $T$ be a complex torus, and let $X \subset T$ be a closed complex submanifold which does not contain any translate of a subtorus of $T$. Then $X$ is Kobayashi hyperbolic.

Proof. Assume $X$ is not Kobayashi hyperbolic. Then there exists a non constant holomorphic map $f: \mathbb{C} \rightarrow X$, and we may assume by Remark 1.17 that $\|d f\|_{h} \leq 1$ for any Hermitian metric $h$ on $X$. Take a metric on $X$ which is the restriction of a metric on $T$ which is itself induced by a constant metric on the universal cover $\mathbb{C}^{n}$ of $T$. Then the map $f$ lifts to a holomorphic map $\tilde{f}: \mathbb{C} \rightarrow \mathbb{C}^{n}$ which has bounded differential, hence must be affine linear. It follows that the closure (for the real topology or analytic Zariski topology) of $\operatorname{Im} f$ is a translate of a subtorus of $T$ which is contained in $X$.

Coming back to Brody's proof of the existence of entire curves in non Kobayashi hyperbolic compact complex manifolds, we observe that it does not give any precision about where the curve is. For example, assume that the sequence $\phi_{n}(0)$ converges to a point $x \in X$, where $\phi_{n}$ is as in the statement of Brody's theorem, (we will say then that the Kobayashi infinitesimal pseudometric of $X$ is degenerate at the point $x$ ). Then it does not follow that one can construct $f$ such that $x$ lies in the closure of the image of $f$. Looking a little more precisely at the proof, we can however prove the following :

Proposition 1.19. Assume that $X$ is compact and that the Kobayashi infinitesimal pseudometric of $X$ is degenerate at the point $x$. Then there exists a non constant holomorphic map

$$
f: \mathbb{C} \rightarrow X,
$$


such that $x$ satisfies

$$
d_{K, X}(x, u)=0, \forall u \in \operatorname{Im} f .
$$

Corollary 1.20. Let $X$ be compact. Then for any $x \in X$, the set

$$
Z_{x}:=\left\{y \in X, d_{K, X}(x, y)=0\right\}
$$

is reduced to $\{x\}$ or contains $\operatorname{Im} f$ for a non constant holomorphic map $f$ from $\mathbb{C}$ to $X$ (that is an entire curve).

Proof of Corollary. Indeed, if $Z_{x}$ contains a point $y \neq x$, it follows that the infinitesimal pseudometric has to be degenerate at $x$, since otherwise, by Royden's theorem, there is an estimate

$$
d\left(x, x^{\prime}\right) \geq c d_{h}\left(x, x^{\prime}\right)
$$

for $x^{\prime}$ in a small neighbourhood of $x$, hence a lower bound

$$
d\left(x, x^{\prime}\right) \geq \operatorname{Min}\left(\epsilon, c d_{h}\left(x, x^{\prime}\right)\right)
$$

for any $x^{\prime}$.

Proof of Proposition 1.19. We observe the following: Lemma 1.16 introduces reparametrizations

$$
\psi_{n}=\phi_{n} \circ t_{n} \circ g_{n}
$$

of the initial sequence $\phi_{n}$. There are now two possibilities :

1) We have $\lim _{t \rightarrow \infty} t_{n}=0$ : we know that $\lim _{n \rightarrow \infty} \phi_{n}(0)=x$. We may assume that the sequence $\psi_{n}(0)$ converges to $f(0)$, so it suffices to show that

$$
\lim _{n \rightarrow \infty} d_{K, X}\left(\psi_{n}(0), \phi_{n}(0)\right)=0 .
$$

Now we have $\psi_{n}(0)=\phi_{n} \circ t_{n}\left(y_{n}\right)$ and so the result follows from the inequality $d_{K, X}\left(\phi_{n} \circ t_{n}\left(y_{n}\right), \phi_{n}(0)\right) \leq d_{P}\left(t_{n} y_{n}, 0\right)$ and

$$
\lim _{n \rightarrow \infty} d_{P}\left(t_{n} y_{n}, 0\right)=0 \text {. }
$$

2) We have $\lim _{t \rightarrow \infty} t_{n} \neq 0$ : this case is still simpler. Indeed, in this case, instead of considering the maps $\psi_{n}$, we can consider the maps $\chi_{n}=\phi_{n} \circ t_{n}$ from $D_{r_{n}}$ to $X$. They form an equicontinuous family which satisfies :

$$
\lim _{n \rightarrow \infty} \chi_{n}(0)=x, \liminf _{n \rightarrow \infty}\left|d \chi_{n}(0)\right| \neq 0 .
$$

Hence we can extract a subsequence converging uniformly on compact sets to a non constant holomorphic map $f: \mathbb{C} \rightarrow X$ satisfying $f(0)=x$. 
Another important application of Brody's Lemma is the following theorem.

TheOREm 1.21. Let $f: \mathcal{X} \rightarrow B$ be a proper holomorphic map. Assume that a fiber $X_{0}$ is Brody hyperbolic. Then there is an open neighborhood $U$ of 0 such that all fibers $X_{t}, t \in U$ are Brody hyperbolic. In particular, Kobayashi hyperbolicity is an open property for compact complex manifolds (or analytic spaces).

Here, for a complex analytic space $Z$, being "Brody hyperbolic" means that there is no non constant holomorphic map from $\mathbb{C}$ to $Z$.

Proof. Indeed, choose a metric on $\mathcal{X}$ and assume to the contrary that there is a sequence of points $t_{n} \in B$ tending to 0 , with $X_{t_{n}}$ not hyperbolic. By Theorem 1.15 and properness of $f$, there exist holomorphic maps $\phi_{n}: \mathbb{C} \rightarrow X_{t_{n}}$ satisfying

$$
\sup _{u \in \mathbb{C}}\left\|d \phi_{n}(u)\right\|=\left\|d \phi_{n}(0)\right\|=1 .
$$

By equicontinuity, using properness of $f$, passing to a subsequence if necessary, these maps converge uniformly to a non constant holomorphic map from $\mathbb{C}$ to $X_{t_{0}}$, which is a contradiction.

In the non compact case, Theorem 1.15 is not true anymore. However, for hypersurface complements, the following is true :

THEOREM 1.22. Let $X$ be compact complex manifold and $Y \subset X$ be an hypersurface. If $Y$ is Brody hyperbolic and $U:=X \backslash Y$ is Brody hyperbolic, then $U$ is Kobayashi hyperbolic.

The proof uses Brody's reparameterization Lemma for holomorphic maps $\phi: \Delta \rightarrow U$. The point is to show that if a sequence of such maps has an unbounded differential at 0 , after rescaling and reparameterization, one can extract a subsequence converging to a non constant holomorphic map with value either in $U$ or in $Y$.

1.1.4. Relation between volume and metric. It is natural to try to establish relations between the various $\Psi_{X}^{p}$. The only obvious fact is the following :

Lemma 1.23. Assume $\Psi_{X}$ vanishes at $x$, or more generally that there is a sequence $x_{n}$ of points of $X$ such that $\lim _{n \rightarrow \infty} x_{n}=x$ and $\lim _{n \rightarrow \infty} \Psi_{X}\left(x_{n}\right)=0$. Then the Kobayashi pseudo-metric is degenerate at $x$.

Proof. By assumption, there exists a sequence of holomorphic maps

$$
\phi_{k}: D^{n} \rightarrow X
$$


such that

$$
\phi_{k}(0)=x_{k}, \lim _{k \rightarrow \infty}\left|J_{\phi_{k}}(0)\right|=\infty .
$$

Here the Jacobian determinant $J_{\phi_{k}}$ is computed via a choice of holomorphic coordinates on $X$, and with respect to the natural coordinates of $D^{n}$ at 0 .

Now, if $\lim _{k \rightarrow \infty}\left|J_{\phi_{k}}(0)\right|=\infty$, at least one of the colums vectors $u_{k}^{i}$ of the Jacobian matrix has a modulus which tends to infinity, with respect to a fixed Hermitian metric $h$ on $X$. But this column vector is $f_{k *}\left(\frac{\partial}{\partial t}\right)$ for some holomorphic map $f_{k}: D \rightarrow X$, such that $f_{k}(0)=x_{k}$. Hence the Kobayashi infinitesimal pseudo-metric is degenerate at $x$.

It seems very natural to ask conversely :

Conjecture 1.24. Assume that $d_{K, X}^{\text {inf }}$ is degenerate everywhere on $X$. Does it follow that $\Psi_{X}=0$ almost everywhere?

We shall come back to a similar problem in section 2.1.3.

1.2. Curvature arguments. We explain here a few basic arguments from analysis, which give sufficient criteria for measure or distance hyperbolicity. In particular we explain the proof of the GriffithsKobayashi theorem on the measure-hyperbolicity of varieties of general type.

1.2.1. Hyperbolic geometry and the Ahlfors-Schwarzlemma. AhlforsSchwarz Lemma (Proposition 1.25 below) is the best known tool to relate curvature properties of a complex manifold and holomorphic maps from disks or polydisks to it. Recall the Poincaré Kähler form

$$
\Omega_{1}=\frac{i}{2} \frac{d z \wedge d \bar{z}}{\left(1-|z|^{2}\right)^{2}}
$$

on the disk $D$. There is the associated product Kähler form

$$
\Omega=\sum_{j=1}^{j=n} \frac{i}{2} \frac{d z_{j} \wedge d \overline{z_{j}}}{\left(1-\left|z_{j}\right|^{2}\right)^{2}}
$$

on the polydisk $D^{n}$. The associated volume form is the hyperbolic volume form

$$
\kappa_{n}=\frac{\Omega^{n}}{n !}=\frac{i^{n}}{2^{n}} \wedge_{j=1}^{j=n} \frac{d z_{j} \wedge d \overline{z_{j}}}{\left(1-\left|z_{j}\right|^{2}\right)^{2}} .
$$

The Ricci form $i \partial \bar{\partial} \log \kappa_{n}$ is equal to

$$
-2 i \sum_{j} \partial \bar{\partial} \log \left(1-\left|z_{j}\right|^{2}\right)=2 i \sum_{j} \partial\left(\frac{z_{j}}{1-\left|z_{j}\right|^{2}} d \overline{z_{j}}\right)
$$




$$
=2 i \sum_{j} \frac{d z_{j} \wedge d \overline{z_{j}}}{\left(1-\left|z_{j}\right|^{2}\right)^{2}}=4 \Omega .
$$

Hence we conclude that $\kappa_{n}$ satifies the conditions :

$$
i \partial \bar{\partial} \log \kappa_{n}>0
$$

$$
\left(i \partial \bar{\partial} \log \kappa_{n}\right)^{n}=4^{n} n ! \kappa_{n} .
$$

We shall denote by $c_{n}$ the constant $4^{n} n$ !. Ahlfors-Schwarz lemma, or rather its $n$-dimensional version, says the following :

Proposition 1.25. Let $\mu$ be a mild pseudo-volume form on $D^{n}$ which satisfies the conditions

$$
\begin{array}{r}
i \partial \bar{\partial} \log \mu>0, \\
(i \partial \bar{\partial} \log \mu)^{n} \geq c_{n} \mu .
\end{array}
$$

Then

$$
\kappa_{n} \geq \mu \text {. }
$$

Here a mild pseudo-volume form is a pseudo-volume form which can be locally written as $|g|^{\frac{1}{r}} \psi$ for some holomorphic function $g$, some non negative integer $r$ and $C^{\infty}$ volume form $\psi$. In other words, it vanishes mildly where it vanishes. Its Ricci form $i \partial \bar{\partial} \log \mu$ is defined as $i \partial \bar{\partial} \log \psi$ in the above local form.

Proof. The proof is well known. It ultimately relies on the maximum principle. One first replaces $D^{n}$ by $D_{1-\epsilon}^{n}$, and $\kappa_{n}$ by the Poincaré volume form $\kappa_{n, \epsilon}$. Proving the inequality (1.8) for $\kappa_{n, \epsilon}$ on $D_{1-\epsilon}^{n}$ will give the result since $\kappa_{n, \epsilon}$ converges to $\kappa_{n}$ uniformly on compact sets of $D^{n}$ when $\epsilon$ tends to 0 .

Next one observes that the function $f=\frac{\mu}{\kappa_{n}}$ is continuous and vanishes on the boundary of $D_{1-\epsilon}^{n}$ and on the singular locus of $\mu$. Hence it has a maximum in $D_{1-\epsilon}^{n}$, and if $x$ is a point where $f$ is maximum, $f$ is non zero and $C^{\infty}$ at $x$. Assume by contradiction that $f(x)>1$. Then we have

$$
\mu>\kappa_{n}
$$

at $x$. Using (1.5) and (1.7) it follows that

$$
(i \partial \bar{\partial} \log \mu)^{n}>\left(i \partial \bar{\partial} \log \kappa_{n}\right)^{n}
$$

at $x$ or

$$
(i \partial \bar{\partial} \log \mu)^{n}-\left(i \partial \bar{\partial} \log \kappa_{n}\right)^{n}>0 .
$$

But this expression can be written as

$$
(i \partial \bar{\partial} \log f) \Theta>0
$$


where, using the conditions (1.4) and (1.6), the $(n-1, n-1)$-form $\Theta$ is positive.

It follows that the $(1,1)$-form $i \partial \bar{\partial} \log f$ cannot be semi-negative at $x$, contradicting the fact that $f$ has a maximum here.

There are a number of applications of Ahlfors-Schwarz lemma. We state here the most direct ones.

THEOREM 1.26. (Kobayashi) The Kobayashi pseudo-volume form $\Psi_{D^{n}}$ is equal to $\kappa_{n}$. In particular, for $n=1$ the Kobayashi pseudodistance $d_{K, D}$ is equal to the Poincaré distance $d_{P}$.

Proof. We use the fact that $A$ ut $D^{n}$ acts transitively on $D^{n}$. Let now $\psi \in$ Aut $D^{n}$. Applying Ahlfors-Schwarz Lemma to $\mu=\psi^{*} \kappa_{n}$ gives $\psi^{*} \kappa_{n} \leq \kappa_{n}$, and applying it to $\psi^{-1}$, we conclude that $\psi^{*} \kappa_{n}=\kappa_{n}$. From the definition of $\Psi$, the fact that $A u t D^{n}$ acts transitively, and the equality above, we now conclude that $\Psi_{D^{n}} \leq \kappa_{n}$.

Next, if $\phi: D^{n} \rightarrow D^{n}$ is a holomorphic map which is generically of maximal rank, we apply Ahlfors-Schwarz lemma to $\mu=\phi^{*} \kappa_{n}$, which gives

$$
\phi^{*} \kappa_{n} \leq \kappa_{n}
$$

By the definition of $\Psi$, this implies that

$$
\Psi_{D^{n}} \geq \kappa_{n}
$$

The next immediate application concerns projective varieties with curvature conditions :

THEOREM 1.27. Let $X$ be a compact complex manifold with $\Omega_{X}$ ample. Then $X$ is Kobayashi hyperbolic.

Recall that a vector bundle $E$ on $X$ is said to be ample if the line bundle $\mathcal{O}_{\mathbb{P}\left(E^{*}\right)}(1)$ is ample on $\mathbb{P}\left(E^{*}\right)$.

Proof. The assumption implies the existence of a Hermitian metric $h$ on the line bundle $\mathcal{O}_{\mathbb{P}\left(T_{X}\right)}(-1)$ on the projective bundle $\pi: \mathbb{P}\left(T_{X}\right) \rightarrow$ $X$, which satisfies the condition that $i \partial \bar{\partial} \log h>0$. After rescaling if necessary, one may assume that $h$ satisfies the following inequality, for any tangent vector $u \in T_{\mathbb{P}\left(T_{X}\right), x}$ such that $\pi_{*} u=x$ :

$$
i \partial \bar{\partial} \log h(u, \bar{u}) \geq 4 h(x) \text {. }
$$

(In this inequality, as in the condition $\pi_{*} u=x$, we have furthermore chosen a lift of $x$ to some point in $T_{X}$.) 
For any non constant holomorphic map $f: D \rightarrow X$, with tangent lift $\tilde{f}: D \rightarrow \mathbb{P}\left(T_{X}\right), h$ provides a metric on $\tilde{f}^{*} \mathcal{O}_{\mathbb{P}\left(T_{X}\right)}(-1)$, hence a pseudo-metric $\tilde{f}^{*} h$ on $T_{D}$, using the natural map $T_{D} \rightarrow \tilde{f}^{*} \mathcal{O}_{\mathbb{P}\left(T_{X}\right)}(-1)$. Let $\omega_{\tilde{f}^{*} h}$ be the associated semi-positive $(1,1)$-form on $D$. Then $(1.9)$ gives :

$$
\tilde{f}^{*} i \partial \bar{\partial} \log h \geq 4 \omega_{\tilde{f}^{*} h} .
$$

Indeed, by definition of the tangent lift, we have everywhere on $D$, the equalities

$$
f_{*}=\pi_{*} \circ \tilde{f}_{*}, f_{*}=\tilde{f} .
$$

Now if $f: D \rightarrow X$ is a non constant holomorphic map with tangent lifting $\tilde{f}$, the inequality (1.10), where $\tilde{f}^{*} i \partial \bar{\partial} \log h$ is the Ricci form of $\omega_{\tilde{f}^{*} h}$ because $\tilde{f}$ is the tangent lifting, implies by Ahlfors-Schwarz lemma that $\omega_{\tilde{f} * h} \leq \Omega_{1}$. This being true for any holomorphic map from the disk to $X$ says exactly that the infinitesimal pseudo-metric of $X$ satisfies

$$
d_{K, X}^{\text {inf }} \geq|\quad|_{h} .
$$

REMARK 1.28. The last inequality is an inequality of Finsler pseudometrics on $T_{X}$, that is of Hermitian pseudo-norms on the line bundle $\mathcal{O}_{\mathbb{P}\left(T_{X}\right)}(-1)$ on $\mathbb{P}\left(T_{X}\right)$.

To conclude this section, we have the following "main Theorem" ([44], [43], [35], [74]) concerning the pseudo-volume forms $\Psi_{X}, \widetilde{\Psi}_{X}$.

THEOREM 1.29. If $X$ is a projective variety of general type, $\widetilde{\Psi}_{X}>0$ on a Zariski dense open set of $X$.

REMARK 1.30. The proof shows in fact that $\widetilde{\Psi}_{X} \geq \mu$ for some mild pseudo-volume form $\mu$ on $X$.

Proof. Let us restrict first for simplicity to working with $\Psi_{X}$ and in the case where the canonical bundle of $X$ is ample (this is the case considered by Griffiths). In this case, there exists a volume form $\mu$ on $X$ which satisfies $i \partial \bar{\partial} \log \mu>0$. Rescaling, and using compactness, we may assume that

$$
i \partial \bar{\partial} \log \mu>0,(i \partial \bar{\partial} \log \mu)^{n} \geq c_{n} \mu .
$$

If $\phi: D^{n} \rightarrow X$ is holomorphic and generically of maximal rank $n=$ $\operatorname{dim} X$, we can then apply Ahlfors-Schwarz lemma to $\phi^{*} \mu$, which gives

$$
\phi^{*} \mu \leq \kappa_{n} .
$$


By the definition of $\Psi_{X}$, this implies that

$$
\Psi_{X} \geq \mu \text {. }
$$

In the general case, one notes that $X$ being of general type, for any given ample line bundle $L$ on $X$, there is a non zero section $\sigma$ of $K_{X}^{\otimes r} \otimes$ $L^{-1}$, that is an inclusion $L \hookrightarrow K_{X}$. Thus any Hermitian metric $h$ on $L^{-1}$ will provide a mild pseudo-volume form $\mu=h^{\frac{1}{r}}$, with degeneracy locus equal to the divisor of $\sigma$. Choosing $h$ such that $i \partial \bar{\partial} \log h>0$, we then conclude as above.

1.2.2. Another definition of the Kobayashi infinitesimal pseudo-metric. It is interesting, having in mind to relate algebraic geometry and analytic geometry as will be done at least conjecturally in next chapter, to note that Demailly, Lempert, and Shiffman $[\mathbf{2 3}]$ have an alternative way to compute the Kobayashi infinitesimal pseudo-metric for projective varieties, which uses only algebraic curves, together with their Poincaré metric. This is based on their approximation theorem, which is stated below.

Definition 1.31. A holomorphic map $\phi: D \rightarrow X$ is said to be Nash algebraic, if there exist an algebraic curve $C$, and a correspondence

$$
f: \begin{gathered}
C \\
r \downarrow \\
\\
\mathbb{P}^{1}
\end{gathered}
$$

such that $r^{-1}(D)$ has one component $D^{\prime}$ which is isomorphic to $D$ via $r$, and

$$
\phi=f \circ r_{\mid D^{\prime}}^{-1}
$$

Here we see $D$ as naturally imbedded in $\mathbb{P}^{1}$. The theorem of $[\mathbf{2 3}]$, (which we state here only for the disk) is the following :

TheOREM 1.32. Any holomorphic map $\phi: D \rightarrow X$, where $X$ is projective, can be approximated uniformly on compact sets by Nash algebraic maps. Furthermore we can approximate $f$ by Nash algebraic maps $g$ such that $g(0)=f(0)$ and $f_{*}$ is proportional to $g_{*}$ at 0 .

As a corollary we get the following way of computing the Kobayashi infinitesimal pseudo-metric of a projective complex manifold. The Poincaré metric \|\|$\|_{P}$ on the disk $D$ induces the Poincaré metric \|\|$_{P, C}$ on each smooth curve $C$ of genus $g \geq 2$, since $D$ is the universal cover of $C$, and $A$ ut $D$ acts preserving \|\|$_{P}$. We have the following (see [23]: 
Proposition 1.33. For $x \in X, u \in T_{X, x}$ we have

$$
d_{K, X}^{i n f}(u)=\inf _{C, \phi, v}\|v\|_{P, C},
$$

where $C$ is a smooth projective curve, $\phi: C \rightarrow X$ is a morphism, $v \in T_{C, c}, \phi(c)=x, \phi_{*}(v)=u$.

Here we make the convention that $\|v\|_{P, C}=0$ if $C$ has genus $\leq 1$.

Proof. The inequality $\leq$ is immediate. Indeed, if $C, \phi$ are as above, let $s: D \rightarrow C$ be the universal covering map, and let $\psi=\phi \circ s$. We may assume that $s(0)=c$ using the fact that Aut D acts transitively on $D$. We have $\psi_{*}\left(\frac{\partial}{\partial t}\right)=\lambda u$, and since $\phi_{*}(v)=u$, we have $s_{*}\left(\lambda^{-1} \frac{\partial}{\partial t}\right)=v$. Hence $|\lambda|$ satisfies

$$
\|v\|_{P, C}=\left\|\lambda^{-1} \frac{\partial}{\partial t}\right\|_{P}=\left|\lambda^{-1}\right| .
$$

By the definition of the Kobayashi infinitesimal pseudo-metric, we then conclude that

$$
\|v\|_{P, C} \geq d_{K, X}^{i n f}(u) .
$$

In the other direction, let $x \in X, u \in T_{X, x}$ and let $\phi: D \rightarrow X$ be a holomorphic map, with $\phi(0)=x, \phi_{*}\left(\frac{\partial}{\partial t}\right)=\lambda u$. We know by Theorem 1.32 that $\phi$ can be approximated uniformly on compact sets by Nash algebraic maps $f \circ r^{-1}$, where $f: C \rightarrow X$ is a morphism from an algebraic curve $C, f(c)=x, r(c)=0$ and $\left(f \circ r^{-1}\right)_{*}, \phi_{*}$ are proportional at 0 . We identify via $r$ the disks $D^{\prime}$ and $D$. We have $f_{*}\left(\frac{\partial}{\partial t}\right)=(\lambda+\epsilon) u$, where $\epsilon$ can be assumed to be arbitrarily small. By Ahlfors-Schwarz Lemma 1.25, we have

$$
\|\|_{P, C \mid D} \leq\|\|_{P}
$$

It follows that

$$
\left\|\frac{\partial}{\partial t}\right\|_{P, C} \leq 1
$$

hence, since $v=\frac{1}{\lambda+\epsilon} \frac{\partial}{\partial t}$, we have

$$
\|v\|_{P, C} \leq\left|\frac{1}{\lambda+\epsilon}\right| \leq \frac{1}{|\lambda|-|\epsilon|} .
$$

Since $d_{K, X}^{\text {inf }}(u)$ is the inf of the $\frac{1}{|\lambda|}$ for $\lambda$ as above, the inequality $\geq$ is proved. 
1.2.3. Jet differentials. The most immediate application of AhlforsSchwarz Lemma 1.25 (case $n=1$ ) to the Kobayashi pseudo-metric is Theorem 1.27. This theorem uses unfortunately very strong assumptions, which are not satisfied in many cases where it is conjectured, and even sometimes proved, that the variety is Kobayashi hyperbolic, for example high degree hypersurfaces in projective space (see section 3.1.3).

Green and Griffiths [31] show how to apply Ahlfors-Schwarz Lemma to much softer objects than Hermitian metrics, namely jet pseudometrics, which led to important applications (Theorems 2.7, 3.14, 3.17). The general idea is as follows : for a complex manifold $X$, consider the jet space $J_{k}(X)$ which is the space of morphisms

$$
\phi: \operatorname{Spec} \mathbb{C}[t] /\left(t^{k+1}\right) \rightarrow X,
$$

or order $k$ jets of germs of holomorphic maps $D \rightarrow X$ defined near 0 . This space is a vector bundle over $X$ of $\operatorname{rank} n k, n=\operatorname{dim} X$, as one sees by taking local coordinates on $X$. There is a natural compact version $P_{k}(X)$ of $J_{k}(X)$, which is the quotient of $J_{k}(X) \backslash 0$ - section by the $\mathbb{C}^{*}$-action given by rescaling. The interest of these spaces lies in the fact that any holomorphic map $f: D \rightarrow X$ admits a canonical lift $f_{k}: D \rightarrow J_{k}(X)$, which associates to each point $t$ the $k$-jet of $f$ at $t$. If $f$ is non-constant, there is also a canonical lift $\bar{f}_{k}: D \rightarrow P_{k}(X)$ obtained by projecting the previous one.

$k$-jet differentials of degree $m$ are defined as sections of the bundle $\mathcal{O}_{P_{k}}(m)$, where one sees $P_{k}$ as a weighted projective bundle over $X$. In other words a $k$-jet differential is a polynomial function $\sigma$ on $J_{k}(X)$ which satisfies the condition

$$
\sigma\left(\lambda \cdot f_{k}\right)=\lambda^{m} \sigma\left(f_{k}\right)
$$

where for $\lambda \in \mathbb{C}^{*}, \lambda$. denotes the action of $\lambda$ on $J_{k}(X)$ by rescaling. Jet differentials can be used to define jet pseudo-metrics, which generalize Finsler pseudo-metrics on tangent space. A Finsler pseudo-metric on $T_{X}$ is a function || on $T_{X}$ which satisfies the condition

$$
|\lambda u|=|\lambda||u|, u \in T_{X, x}, \lambda \in \mathbb{C} .
$$

In other words, it is a Hermitian pseudo-norm on the line bundle $\mathcal{O}(-1)$ on $\mathbb{P}\left(T_{X}\right)$. The Kobayashi infinitesimal pseudo-metric is an example.

More generally a $k$-jet pseudo-metric is a function || on $J_{k}(X)$, which satisfies the condition

$$
\left|\lambda \cdot u_{k}\right|=|\lambda|\left|u_{k}\right|, u_{k} \in J_{k}(X), \lambda \in \mathbb{C} .
$$

Equivalently, this is a pseudo Hermitian norm on the line bundle $\mathcal{O}(-1)$ on $P_{k}$. If $\sigma_{1}, \ldots, \sigma_{N}$ are jet differentials of degree $m$ on $P_{k}(X)$, one can 
construct the jet pseudo-metric

$$
\left|f_{k}\right|=\left(\sum_{i}\left|\sigma_{i}\right|^{2}\right)^{\frac{1}{2 m}}
$$

It vanishes on the base-locus of the $\sigma_{i}$ 's. A jet pseudo-metric as above has a curvature on holomorphic disks (along which it is not everywhere degenerate), defined as follows : If $f: D \rightarrow X$ is a holomorphic map, with lift $f_{k}: D \rightarrow J_{k}(X)$, consider the function

$$
\left|f_{k}\right|=\left(\sum_{i}\left|\sigma_{i}\left(f_{k}\right)\right|^{2}\right)^{\frac{1}{2 m}}
$$

on $D$. Assuming it is non zero, the $(1,1)$-form

$$
-i \partial \bar{\partial} \log \left|f_{k}\right|^{2}
$$

is $C^{\infty}$ on $D$ and the curvature will be the ratio

$$
R=-\frac{i \partial \bar{\partial} \log \left|f_{k}\right|^{2}}{\left|f_{k}\right|^{2} i d z \wedge d \bar{z}}
$$

This ratio is a function on $D$. Note that if $f$ is replaced with $f \circ \lambda$ for some $\lambda \in \mathbb{C}^{*}$, the curvature is not changed.

Ahlfors-Schwarz lemma applied to the case $n=1$ shows the following :

LEMMA 1.34. (Green-Griffiths) If for some $A>0$, the jet pseudometric constructed as above has curvature $\leq-A$ on a given disk $f$ : $D \rightarrow X$, then one has

$$
\left|f_{k}\right|^{2} i d z \wedge d \bar{z} \leq 4 A^{-1} \Omega_{1}
$$

where $\Omega_{1}$ is the Kähler form of the Poincaré metric (Definition 1.4).

As a corollary, one gets :

COROllary 1.35. If for a given $A>0$ the jet pseudo-metric constructed as above has curvature $\leq-A$ on disks whose lifts are not contained in a given proper algebraic subset $B \subset J_{k}(X)$, then any holomorphic map $f: \mathbb{C} \rightarrow X$ must have its canonical lift contained in $B$.

Indeed, we apply the lemma to disks contained in $\mathbb{C}$ and centered at 0 , noting that the Poincaré metric \|\|$_{P}$ on them converges uniformly on compact sets to 0 , when the radius tends to infinity.

Green and Griffiths deduce from this : 
TheOREM 1.36. Assume that $L$ is an ample line bundle on $X$, and that

$$
\sigma \in H^{0}\left(P_{k}, \mathcal{O}_{P_{k}}(m) \otimes \pi^{*} L^{-1}\right)
$$

where $\pi: P_{k} \rightarrow P$ is the structural map. Then for any non constant holomorphic map $f: \mathbb{C} \rightarrow X, \sigma$ vanishes on the lift $\bar{f}_{k}$.

Assume for simplicity that $L$ is very ample, and let $\alpha_{i}, i=1, \ldots, N$ be a basis of $H^{0}(X, L)$. The theorem is proved by computing the sectional curvature of the jet pseudo-metric associated to the degree $m$ jet differentials $\sigma \alpha_{i}$.

Finally a Riemann-Roch estimate shows the following :

THEOREM 1.37. (Green-Griffiths) If $X$ is a surface of general type, and $L$ is any ample line bundle, there exists a non-zero $k$-jet differential of degree $m$, for $m, k$ large enough.

REMARK 1.38. Instead of $k$-jet differentials for possibly large orders $k$, one could content ourselves with sections of $S^{m} \Omega_{X}$, which are degree $m$ 1-jet differentials. This is done by Bogomolov (see Theorem 2.6) in a more algebraic context where algebraic curves are studied, and McQuillan (Theorem 2.7) to study entire curves on surfaces. The advantage of doing this is that one is looking at first order jets, so that in this case, the proper subset $B$ in Theorem above can be seen as a multifoliation on $X$. The disadvantage however comes from the fact that the Riemann-Roch estimate works then only if $c_{1}(X)^{2}>c_{2}(X)$, an assumption which is not satisfied eg by surfaces in $\mathbb{P}^{3}$. A different notion of jet differentials allows Demailly and Elgoul to have a better Riemann-Roch estimate, working for high degree surfaces in $\mathbb{P}^{3}$, (see section 3.1.3).

\subsection{Conjectures, examples.}

1.3.1. Kobayashi's conjecture on volumes. There are several versions of a possible converse to the Griffiths-Kobayashi-Ochiai Theorem 1.29. The most ambitious one is the following :

Conjecture 1.39. Let $X$ be a smooth projective manifold which is not of general type. Then $\Psi_{X}=0$ on a dense Zariski open set of $X$.

The following example provides evidences for this :

LEMMA 1.40. [31] If $X$ is either uniruled, or rationally swept out by elliptic curves, then $X$ satisfies conjecture 1.39 .

Recall that $X$ is uniruled if there exists a dominant rational map

$$
\phi: Y \times \mathbb{P}^{1} \rightarrow X,
$$


where $\operatorname{dim} Y=n-1, n=\operatorname{dim} X$, and that $X$ is rationally swept out by elliptic curves if there exists a dominant rational map

$$
\phi: E \rightarrow X,
$$

where $E$ is a $n$-dimensional variety which admits a fibration $\pi: E \rightarrow$ $Y$ over a smooth quasi-projective variety $Y$, whose fiber is a smooth elliptic curve.

Proof. In both case the indeterminacy locus of $\phi$ is of codimension $\geq 2$, hence does not meet the generic fiber of $p r_{1}$ in the first case, or $\pi$ in the second case. So there is a dense Zariski open set $U \subset Y$ over which $\phi$ is defined. Denote by $\phi_{U}$ the restriction of $\phi$ to $U \times \mathbb{P}^{1}$ or to $E_{U}:=\pi^{-1}(U)$ in the second case. Then the decreasing volume property says that

$$
\phi_{U}^{*} \Psi_{X} \leq \Psi_{U \times \mathbb{P}^{1}}
$$

and we have the similar inequality in the second case. But it is easy to see that for $E_{U}$ and for $U \times \mathbb{P}^{1}$, the Kobayashi-Eisenman pseudo-volume form vanishes. Indeed, for the last one, this follows from Lemma 1.12, and for the first one, it suffices to show that for small open sets $V \subset U$, the Kobayashi-Eisenman pseudo-volume forms of $E_{V}$ vanishes. But for small $V$, the universal cover of $E_{V}$ is isomorphic to $V \times \mathbb{C}$, so again Lemma 1.12 applies.

It follows that in both cases we have

$$
\phi_{U}^{*} \Psi_{X}=0
$$

and this implies that $\Psi_{X}=0$ on $\operatorname{Im} \phi_{U}$ away from the branch locus of $\phi$.

Yau's variant (Definition 1.13) of the Kobayashi-Eisenman pseudometrics allows to weaken conjecture 1.39 as follows :

CONJECTURE 1.41. Let $X$ be a smooth projective manifold which is not of general type. Then $\widetilde{\Psi}_{X}=0$ on a dense Zariski open set of $X$.

We have then the following example, which generalizes the previous ones :

Lemma 1.42. Assume there exist a family $\pi: \mathcal{A} \rightarrow Y$ of smooth abelian varieties, and a dominating rational map

$$
\phi: \mathcal{A} \rightarrow X,
$$

which is non constant on the generic fiber of $\pi$. Then $\widetilde{\Psi}_{X}=0$ on a dense Zariski open set of $X$. 
Proof. Let $n=\operatorname{dim} X$. The Kobayashi-Eisenman-Yau $n$-pseudovolume form $\widetilde{\Psi}_{\mathcal{A}}^{n}$ is equal to $\pi^{*} \widetilde{\Psi}_{Y}^{n}$, as shows the local uniformisation of $\mathcal{A}$ over $Y$ by products $V \times \mathbb{C}^{r}$. The decreasing volume property says that

$$
\phi^{*} \widetilde{\Psi}_{X} \leq \widetilde{\Psi}_{\mathcal{A}}^{n},
$$

where $\phi$ is defined. Hence we have the inequality

$$
\phi^{*} \widetilde{\Psi}_{X} \leq \pi^{*} \widetilde{\Psi}_{Y}^{n},
$$

where $\phi$ is defined. But this inequality clearly implies that $\widetilde{\Psi}_{X}=0$ at any point $x \in X$ such that, for some point $y \in \mathcal{A}$ where $\phi$ is defined, $\phi(y)=x, \phi$ is of maximal rank $n$, and $\phi_{*}$ does not vanish identically in the vertical tangent space of $\pi$. It is easy to see that the set of such $x$ 's contains a Zariski open set of $X$, which by assumption is non-empty, hence dense.

Another case where conjecture 1.41 can be checked is the following

Lemma 1.43. Let $X$ be a $K$-trivial projective manifold. Assume that $X$ admits a rational map

$$
\phi: X \rightarrow X
$$

which is of degree $d>1$. Then $\widetilde{\Psi}_{X}=0$ away from the indeterminacy locus of $\phi$.

Proof. Let $\eta$ be a generator of $H^{0}\left(X, K_{X}\right)$. Then $\phi^{*} \eta \in H^{0}\left(X, K_{X}\right)$ must be proportional to $\eta$ :

$$
\phi^{*} \eta=\lambda \eta, \lambda \in \mathbb{C} .
$$

Hence

$$
\phi^{*}(\eta \wedge \bar{\eta})=|\lambda|^{2} \eta \wedge \bar{\eta} .
$$

Integrating both sides over $X$, we conclude that $|\lambda|^{2}=d>1$. Now there exists a complex valued function $\chi$ on $X$ which is uppersemicontinuous and such that

$$
\widetilde{\Psi}_{X}=\chi \eta \wedge \bar{\eta}
$$

Combining the inequality

$$
\phi^{*} \widetilde{\Psi}_{X} \leq \widetilde{\Psi}_{X}
$$

which is true away from the indeterminacy locus of $\phi$ and the equality

$$
\phi^{*}(\eta \wedge \bar{\eta})=d \eta \wedge \bar{\eta}
$$

we now conclude that

$$
d\left|\phi^{*} \chi\right| \leq|\chi|
$$


away from the indeterminacy locus of $\phi$. But this easily implies that $|\chi|=0$ away from the indeterminacy locus of $\phi$ since $\chi$ is bounded.

This example leads to the following question which was asked to me by K. Amerik and S. Cantat (see also [55] for a similar question in the case of $K 3$ surfaces) : (a negative answer would provide a deceptively simple proof of Kobayashi's conjecture 1.41 for Calabi-Yau varieties!)

Question. Are there $K$-trivial varieties which do not admit a rational self-map of degree $>1$ ?

Here are examples of such rational self-maps :

Example 1.44. [67] Consider the Fano variety of lines $F$ of a cubic fourfold $X \subset \mathbb{P}^{5}$. This is a holomorphically symplectic variety, but using higher dimensional cubics, one can construct as well Calabi-Yau similar examples, that is $K$-trivial varieties $Z$ satisfying the vanishing condition $h^{i}\left(\mathcal{O}_{Z}\right)=0,0<i<\operatorname{dim} Z$.

These varieties always admit a self-rational map of degree $>1$. The construction is as follows : if $l \in F$ is a generic line in $X$, there is a unique plane $P \subset \mathbb{P}^{5}$ which is everywhere tangent to $X$ along $l$. The intersection $P \cap X$ equals $2 l+l^{\prime}$ for another line $l^{\prime} \subset X$. The rational map will be $l \mapsto l^{\prime}$. It has degree 16 .

EXAMPLE 1.45. If $X$ is a projective variety which admits a rational fibration

$$
\pi: X \rightarrow-\rightarrow
$$

whose generic fiber is an abelian variety, $X$ admits a self-rational map of degree $d>1$. Indeed, let $L$ be an ample line bundle on $X$. We construct $\phi$ as follows : for $x \in X$ generic, let $A$ be the fiber $\pi^{-1}(\pi(x))$. Let $N:=\operatorname{deg}\left(L_{\mid A}\right)^{2} \in \mathbb{N}^{*}$, where $\left(L_{\mid A}\right)^{2}$ is a zero cycle of $A$ which is well defined up to rational equivalence. To $x, \phi$ associates the unique point $y \in A$ such that

$$
A l b_{A}\left(\left(L_{\mid A}\right)^{2}-(N-1) x-y\right)=0,
$$

where $A l b_{A}$ is the Albanese map of $A$, which is well defined on cycles of degree 0 on $A$. This map has degree $(N-1)^{2 a}$, where $a$ is the dimension of the fibres $A_{y}, y \in Y$. Indeed, it is essentially multiplication by $-(N-1)$ on these fibers. However it is important to note that one does not need a 0 -section in order to define it.

Note that there are examples of varieties which are not of general type but do not admit a self rational map of degree $d>1$. Indeed, let $S$ be a $K 3$ surface which is a double cover of $\mathbb{P}^{2}$ ramified along a smooth 
sextic curve. Let $C \rightarrow C^{\prime}$ be an étale double cover, where $C^{\prime}$ is a curve which admits no other automorphism than the identity. Denote by $i$ the involution acting on $S$ and by $j$ the involution acting on $C$ over $C^{\prime}$. Let $X:=S \times C /(i, j)$. Since $g\left(C^{\prime}\right) \geq 1$, any self rational map $\phi: X \rightarrow X$ should preserve the fibration $\pi: X \rightarrow C^{\prime}$, whose fiber is isomorphic to $S$. Since $A u t C^{\prime}=I d, \phi$ would have in fact to act over $C^{\prime}$. Since the self-rational maps of $S$ form a discrete set, the induced rational map $\phi_{c}: S \rightarrow S$ (which is well defined up to conjugacy by $i$ ) has to be constant. It follows immediately that $\phi$ lifts to $S \times C$ as a product map

$$
\left(\phi_{c}, I d\right)
$$

and that $\phi_{c}$ has to satisfy $i \circ \phi_{c} \circ i=\phi_{c}$. Now it is quite easy to see that $S$ does not admit a self-rational map $\phi_{c}$ of degree $d>1$ satisfying the condition $i \circ \phi_{c} \circ i=\phi_{c}$.

The last weakening of the conjectures above is the following :

Conjecture 1.46. Let $X$ be a projective manifold which is not of general type. Then $\widetilde{\Psi}_{X}=0$ almost everywhere on $X$. Equivalently the associated measure is 0 .

The following example generalizes the one considered previously :

Lemma 1.47. Let $X$ be a projective manifold. Assume that $X$ admits a rational map

$$
\phi: X \rightarrow X
$$

which is of degree $d>1$. Then $\widetilde{\Psi}_{X}=0$ almost everywhere.

Proof. Indeed we know that

$$
\phi^{*} \widetilde{\Psi}_{X} \leq \widetilde{\Psi}_{X} .
$$

Integrating over $X$ gives :

$$
\int_{X} \phi^{*} \widetilde{\Psi}_{X}=d \int_{X} \widetilde{\Psi}_{X} \leq \int_{X} \widetilde{\Psi}_{X}
$$

which gives $\int_{X} \widetilde{\Psi}_{X}=0$ since $d>1$.

1.3.2. Conjectures on metrics. In [42], Kobayashi makes the following conjecture :

Conjecture 1.48. Let $X$ be a $K$-trivial projective variety. Then $d_{K, X}=0$.

REMARK 1.49. The conjecture is stated for those $K$-trivial complex varieties admitting a Ricci flat Kähler metric. By Yau's theorem, this gives all Kähler $K$-trivial compact manifolds. 
Evidences for this conjecture are provided by the following known cases.

EXAMPLE 1.50. Abelian varieties and more generally complex tori satisfy the conjecture, since they are uniformized by affine spaces.

Another known case is the following :

Lemma 1.51. If $X$ is an algebraic $K 3$ surface $S$ or one of its Hilbert schemes $S^{[k]}$ (see $[\mathbf{3}]$ ), then $d_{K, X}=0$.

Proof.It suffices obviously to do the case of $S$. It is known that curves of geometric genus 1 in any complete ample linear system on $S$ form a 1-dimensional family. Heuristically, this fact is proved by a dimension count : if $L$ is an ample line bundle on a $K 3$ surface $S$, with $L^{2}=2 g-2$, one has by Riemann-Roch

$$
h^{0}(S, L)=g+1,
$$

and by adjunction formula, curves $C$ in $|L|$ have genus $g$. So $S$ carry a $g$-dimensional family of curves of genus $g$, and imposing $g-1$ nodes to them provides a 1-dimensional family of curves of geometric genus 1. (Note that one needs actually a supplementary argument to show that the family is not empty. Indeed a similar dimension count could also be done for an abelian surface while the generic abelian surface does not contain elliptic curves.)

Of course, two points $x, y$ lying on such a curve satisfy $d_{K, S}(x, y)=$ 0 . On the other hand, Since the curves are ample, any two such curve meet so have 0 Kobayashi distance. Hence $d_{K, S}=0$.

The next example is more interesting, since it concerns also non hyperkähler varieties.

Lemma 1.52. Let $r: X \rightarrow \mathbb{P}^{n}$ be a double cover ramified along a smooth hypersurface $\Sigma$ of degree $2 n+2$. Then $K_{X}=0$ and $d_{K, X}=0$.

Proof. One notes that $X$ is swept out by elliptic curves. Indeed, consider lines $l \subset \mathbb{P}^{n}$ meeting $\Sigma$ along a 0 -cycle of the form $x+y+z+$ $t+2\left(\sum_{1}^{n-1} x_{i}\right)$. One shows easily that these lines cover $\mathbb{P}^{n}$. But the normalization $\widetilde{E}_{l}$ of the curve $E_{l}:=r^{-1}(l)$ has generically genus 1 .

To conclude the proof, let us for simplicity assume that $n=3$. If $l$ is a line as above, one shows by a cohomological computation that the locus $S_{l}$ in $\mathbb{P}^{3}$ covered by the lines $l^{\prime}$ meeting $l$, is a surface. For any two points $x, y$ of $X$ such that $r(x), r(y) \in S_{l}, d_{K, X}(x, y)=0$ because there are two meeting elliptic curves $E_{x}$ and $E_{y}$ in $X$ passing through $x, y$ respectively. Finally, $S_{l}$ meets any line $l^{\prime}$ as above, because $\operatorname{Pic} X=\mathbb{Z}$ 
so $S_{l}$ is ample, and hence, by the same argument, any point of $X$ is at the 0 Kobayashi distance of $r^{-1}\left(S_{l}\right)$.

The general case will need more steps, introducing $S_{l}^{2}:=$ the set of points through which there is a line $l^{\prime}$ as above meeting $S_{l}$, and so on, until we filled-in $\mathbb{P}^{n}$.

In the opposite direction, Green-Griffiths [31] and later Lang, made a number of conjectures concerning varieties of general type. The most important one might be the following :

Conjecture 1.53. Let $X$ be a projective variety of general type. Then the union of entire curves in $X$ is not Zariski dense.

By Brody's theorem, this is obviously related to the Kobayashi pseudodistance $d_{K, X}$ for such a variety. The following is a stronger form of the Green-Griffiths conjecture.

Conjecture 1.54. Let $X$ be as above. Then the Zariski closure of the set

$$
\left\{(x, y) \in X \times X, x \neq y, d_{K}(x, y)=0\right\}
$$

does not project onto $X$.

It seems that interest in the Kobayashi distance for varieties of general type has more focused on the problem of deciding whether they are Kobayashi hyperbolic or not. We shall come back to this in section 3.1.3, where we shall consider hypersurfaces of degree $d$ in projective space $\mathbb{P}^{n+1}$. Recent progresses show Kobayashi hyperbolicity of generic hypersurfaces for large $d, n$ fixed. However, it seems that nothing is known in the range $n+2<d<2 n$, where the canonical bundle is ample, while $X$ is known to be not Kobayashi hyperbolic.

1.3.3. Campana's construction and conjectures. Campana $[8]$ found recently a very interesting class of varieties enlarging the class of varieties with semi-negative canonical bundles, which he calls "special". Their definition uses the notion of "morphism of general type" introduced by Campana :

Definition 1.55. For $\phi: X \rightarrow Y$ a dominant morphism between smooth projective manifolds, define the discriminant divisor $\Delta \in$ $\operatorname{Pic} Y \otimes \mathbb{Q}$ to be

$$
\Delta=\sum_{i} \alpha_{i} D_{i}
$$

where $D_{i}$ runs over the set of reduced irreducible divisors of $Y$ which have a non reduced inverse image under $\phi$, and $\alpha_{i}$ is determined as $1-\frac{1}{m_{i}}$, where $m_{i}$ is the gcd of the multiplicities of the components of $\phi^{-1}\left(D_{i}\right)$. 
Definition 1.56. A morphism $\phi$ as above is said to be of general type if the $\mathbb{Q}$-divisor $K_{Y}+\Delta$ is big.

Recall that $L$ is said to be "big" if the global sections of $L^{\otimes N}$ for $N$ large enough provide a generically finite rational morphism to projective space.

Definition 1.57. A variety $X$ is said to be of general type if it does not admit a morphism of general type to a positive dimensional variety.

(Here we are giving only a rough version, since the correct final definition will involve rational maps instead of morphisms.)

ExAmple 1.58 . Let $X \rightarrow \mathbb{P}^{r}$ be a family of Calabi-Yau varieties with no multiple fibers. Then $X$ is special.

Campana [8] proves the existence of a universal rational morphism $\phi: X \rightarrow B$ which is of general type and whose fibers are special. He makes the following :

Conjecture 1.59. The Kobayashi pseudodistance $d_{K, X}$ is pulledback from a pseudo-distance on $B$.

\section{Algebraic versions, variants, Lang's conjectures}

2.1. Subvarieties and hyperbolicity. This section is devoted to the description of a number of algebrogeometric substitutes for the analytic notions of hyperbolicity presented in chapter 1. The general idea, due to Lang, is that these analytic properties should be detected via algebraic geometry.

2.1.1. Algebraic hyperbolicity. Let $C$ be a smooth projective curve of genus $g \geq 2$. Then the universal cover of $C$ is the disk $D$, on which the group $\pi_{1}(C)$ acts by automorphisms, which preserve the Poincaré metric (1.3). Hence this metric descends to a metric on $C$, whose Kähler form $\omega_{\text {hyp }}$ is determined by the condition

$$
i \partial \bar{\partial} \log \omega_{\text {hyp }}=4 \omega_{\text {hyp }} .
$$

Note that the left hand side is also $2 \pi$ times the Chern form of the induced metric $\omega_{\text {hyp }}^{-1}$ on the canonical bundle $K_{C}$, hence it is a de Rham representative of the class $c_{1}\left(K_{C}\right)$, so that we have the Gauss-Bonnet formula :

$$
4 \int_{C} \omega_{h y p}=2 \pi(2 g-2) .
$$

One deduces from this : 
Proposition 2.1. [21] Let $X$ be a compact complex manifold which is Kobayashi hyperbolic. Let $h$ be a Hermitian metric on $X$, with corresponding (1,1)-form $\omega_{h}$. Then there exists a constant $A>0$ such that for any non constant holomorphic map

$$
\phi: C \rightarrow X
$$

from a smooth projective curve of genus $g$ to $X$, we have

$$
2 g-2 \geq A \int_{C} \phi^{*} \omega_{h} .
$$

Proof. Since $X$ is Kobayashi hyperbolic and compact, there exists a constant $B$ such that for any $x \in X, u \in T_{X, x}$, we have

$$
d_{K, \text { inf }}(u) \geq B\|u\|_{h} .
$$

By definition of the Kobayashi infinitesimal metric, this means that for any holomorphic map

$$
\psi: D \rightarrow X
$$

we have the inequality

$$
\Omega_{1} \geq B^{2} \psi^{*} \omega_{h}
$$

Since $X$ is Kobayashi hyperbolic, there is no non constant holomorphic map from $\mathbb{P}^{1}$ or from an elliptic curve to $X$. So for any non constant holomorphic map

$$
\phi: C \rightarrow X
$$

we know that $g=g(C) \geq 2$. Let $\pi: D \rightarrow C$ be the universal cover, and let us apply $(2.14)$ to $\psi:=\phi \circ \pi$. Since $\pi^{*} \omega_{\text {hyp }}=\Omega_{1}$, we conclude that

$$
\omega_{\text {hyp }} \geq B^{2} \phi^{*} \omega_{h}
$$

Integrating this inequality over $C$ and applying formula (2.13), we get

$$
\pi(g-1) \geq B^{2} \int_{C} \phi^{*} \omega_{h}
$$

This result led Demailly to the following definition :

Definition 2.2. A compact complex manifold $X$ is said to be algebraically hyperbolic if the conclusion of theorem 2.1 holds, that is, for any Hermitian metric $h$ on $X$ there exists a constant $A$ such that for any holomorphic map

$$
\phi: C \rightarrow X
$$

from a curve of genus $g$ to $X$, we have

$$
2 g-2 \geq A \int_{C} \phi^{*} \omega_{h} .
$$


REMARK 2.3. By compactness of $X$, it suffices to check this for one $h$.

REMARK 2.4. The definition is coherent with other notions of hyperbolicity only for projective varieties. Indeed, general complex tori do not contain any algebraic curve, hence would be algebraically hyperbolic in the above sense! When $X$ is algebraic, one usually chooses for $\omega_{h}$ the Chern form of an Hermitian metric on a ample line bundle $L$ on $X$, so that the integral $\int_{C} \phi^{*} \omega_{h}$ is the degree $\operatorname{deg} \phi^{*} L$.

Proposition 2.1 says that compact Kobayashi hyperbolic complex manifolds are algebraically hyperbolic. The following can be found in $[21]$.

ConjeCture 2.5. For projective complex manifolds, the two notions coincide.

Proposition 1.33 provides some evidence for conjecture 2.5, since it says that for projective $X$, the infinitesimal Kobayashi pseudo-metric can be computed via algebraic curves in $X$. However it just says that if $X$ is not Kobayashi hyperbolic, there is a sequence of triples $\left(C_{n}, x_{n}, \phi_{n}\right)$, where $C_{n}$ is a projective curve, $x_{n}$ is a point of $C_{n}$ and $\phi_{n}$ is a holomorphic map from $C_{n}$ to $X$, such that

$$
\lim _{n \rightarrow \infty}\left\|d \phi_{n, x_{n}}\right\|=\infty,
$$

where the norm is computed w.r.t. the hyperbolic metric on $C_{n}$ and any fixed metric on $X$. The problem is that this is only a pointwise information at $x_{n}$, while in order to prove the conjecture, one would need to have a similar statement either everywhere along $C_{n}$ or in an integrated form.

An earlier important result, where a notion close to algebraic hyperbolicity is implicitely studied, is due to Bogomolov [4]. Some of the arguments introduced in his paper are also useful in the analytic context of entire curves.

THEOREM 2.6. (Bogomolov) Let $S$ be a surface of general type satisfying the condition

$$
c_{1}(S)^{2}>c_{2}(S)
$$

Then for any fixed genus $g$, there are finitely many algebraic varieties parameterizing all curves of geometric genus $g$ on $S$.

Let us sketch the proof. Using Riemann-Roch theorem for the vector bundles $S^{i} \Omega_{S}$ on $S$, inequality (2.15) and the fact that $S$ is of general 
type, imply that for some constant $c>0$, one has the inequality, for all $i \gg 0$ :

$$
H^{0}\left(S, S^{i} \Omega_{S}\right) \geq c i^{3} .
$$

We can view sections of $S^{i} \Omega_{S}$ as sections of the line bundle $\mathcal{O}_{\mathbb{P}}(1)$ on $\mathbb{P}:=\mathbb{P}\left(T_{S}\right)$. Inequality (2.16) then implies that for $i$ large enough, sections of $\mathcal{O}_{\mathbb{P}}(i)$ provide a rational map

$$
\psi: \mathbb{P}\left(T_{S}\right) \rightarrow \mathbb{P}^{N}
$$

which is generically $1-1$ onto its image.

Now let $C \subset S$ be a curve of geometric genus $g$ and $i: \widetilde{C} \rightarrow S$ the natural map, where $\widetilde{C}$ is the normalization of $C$. There is a tangent lifting

$$
j: \widetilde{C} \rightarrow \mathbb{P}\left(T_{S}\right)
$$

of the map $i$, which to a point $c \in \widetilde{C}$ associates the line $i_{*}\left(T_{\widetilde{C}, c}\right)$. The map $j$ satisfies

$$
j^{*}\left(\mathcal{O}_{\mathbb{P}\left(T_{S}\right)}(1)\right)=K_{\widetilde{C}}(-D),
$$

where $D$ is the divisor of $\widetilde{C}$ where $i$ is not an immersion.

Let $B \subset \mathbb{P}\left(T_{S}\right)$ be the subset where $\psi$ is not a morphism, or is not an isomorphism onto its image (i.e. where $\psi^{-1}(\psi(x)) \neq\{x\}$ ). Let us decompose $B$ as $B_{1} \cup B_{2}$, where $B_{1}$ is the union of components not dominating $S, B_{2}$ is the union of components dominating $S$. There are clearly only finitely many curves whose tangent lift lies in $B_{1}$. Next, curves whose tangent lift lies in $B_{2}$ satisfy a first order non linear equation, which becomes linear after passing to the finite covering $B_{2}$. Bogomolov then proves that there are only finitely many families of solutions of such a differential system (see also [38]).

The remaining curves have their tangent lift not contained in $B$. But for such a curve, the map $\psi \circ j$ is generically defined and $1-1$ on its image. On the other hand, relation (2.17) shows that for such curves, we have the inequality

$$
\operatorname{deg} \psi \circ j(\widetilde{C}) \leq 2 g-2 .
$$

One uses then the finiteness of Chow varieties of curves in $\mathbb{P}^{N}$ to conclude.

Much more recently, McQuillan [50] extended such arguments in the context of entire curves, thus proving the Green-Griffiths conjecture for surfaces with $c_{1}^{2}>c_{2}$. 

that

THEOREM 2.7. (McQuillan) Let $S$ be a surface of general type, such

$$
c_{1}(S)^{2}>c_{2}(S)
$$

Then any entire curve in $S$ is algebraically degenerate, i.e. is contained in an algebraic curve $C \subset S$.

The Green-Griffiths conjecture for such surfaces follows :

Corollary 2.8. Let $S$ be as above. Then there are finitely many curves $C \subset S$ such that any non constant holomorphic map from $\mathbb{C}$ to $S$ takes value in one of these curves.

Proof. Indeed, Bogomolov's theorem says that there are finitely many families of rational or elliptic curves on $S$ (that is, curves whose normalization has genus 0 or 1 ). But Lemma 2.19 below shows that rational and elliptic curves do not deform in a surface of general type. Hence there are finitely many rational or elliptic curves.

On the other hand, McQuillan theorem says that entire curves are contained in irreducible curves $C \subset S$, whose normalization must be rational or elliptic. This concludes the proof.

The proof of McQuillan's theorem uses Theorem 1.36 and the computation of the Euler characteristic of $S^{i} \Omega_{S}(-H)$, where $i$ is arbitrarily large, and $H$ is an ample line bundle on $S$. This allows to show the existence of a non-zero section $\sigma$ of this bundle for large $i$. Theorem 1.36 then shows that for any non constant holomorphic map $f: \mathbb{C} \rightarrow S$, its tangent lift

$$
f_{*}: \mathbb{C} \rightarrow \mathbb{P}\left(T_{S}\right)
$$

is contained in the zero locus of $\sigma$.

Now, the heart of the proof of McQuillan is the following see [50] :

THEOREM 2.9. Consider a (maybe singular) algebraic foliation on a surface of general type. Then any parabolic leaf of this foliation is algebraically degenerate.

Here parabolic means uniformized by $\mathbb{C}$. One applies this theorem to the natural foliation on the surface $\Sigma:=V(\sigma)$. (A point of $\Sigma$ over $s \in S$ determines a tangent direction on $S$ at $s$, and one uses the generic isomorphism $T_{\Sigma} \cong \pi^{*} T_{S}$, where $\pi$ is the restriction to $\Sigma$ of the structural morphism, to lift this tangent direction to one on $\Sigma$.) This shows that the tangent lifts of entire curves in $S$ are algebraically degenerate in $\Sigma$, hence that entire curves in $S$ are algebraically degenerate. 
2.1.2. Lang's conjectures. There are two main conjectures by Lang [48] on the subject of entire curves in complex algebraic varieties. We start with the obvious observation that any variety $Y$ which is birationally equivalent to an abelian variety (or more generally a complex torus), is swept out by images of non-constant holomorphic maps

$$
f: \mathbb{C} \rightarrow Y \text {. }
$$

Furthermore, if $Y \stackrel{\psi}{\rightarrow} X$ is a non-constant holomorphic map, the same is true for the variety $\operatorname{Im} \psi$. Lang's amazing conjecture is the following converse to this last statement :

Conjecture 2.10. Let $X$ be a smooth projective variety. Then the union of all non-constant holomorphic maps $f: \mathbb{C} \rightarrow X$ is equal to the union of the images of non-constant rational maps from an abelian variety to $X$.

It would be presumably more reasonable to replace these sets with their analytic or even Zariski closure, but then much of the spirit of this conjecture is lost. Note that Bloch's Theorem says that the conjecture is true if $X$ is a subvariety of an abelian variety.

Remark 2.11. As shown by Cantat $[\mathbf{9}]$, the conjecture cannot be extended to compact complex manifolds, replacing abelian varieties by complex tori. General non algebraic $K 3$ surfaces do not admit any non constant rational map from a complex torus to them. On the other hand, they are not Kobayashi hyperbolic, since this is an open property, and algebraic $K 3$ surfaces are dense in the space of all $K 3$ 's, and they are not Kobayashi hyperbolic (see Lemma 1.51).

The second important conjecture concerns the locus described above (the exceptional locus):

Conjecture 2.12. The exceptional locus is equal to $X$ if and only if $X$ is not of general type.

Note that this last conjecture would imply Kobayashi's conjecture 1.41. Indeed, Kobayashi's conjecture says that if $X$ is not of general type, its pseudo-volume form $\widetilde{\Psi}_{X}$ is equal to 0 on a Zariski open set. On the other hand, if we combine the two conjectures of Lang, we get the following :

Conjecture 2.13. If $X$ is not of general type, the union of the images of non-constant rational maps $\phi: A \rightarrow X$ from a abelian variety to $X$ cover $X$. 
If the conjecture above is true, one uses next a countability argument for families parametrizing abelian varieties $A$ together with non-constant rational map $\phi$ to $X$, and a Baire argument to deduce that there should exist a quasi-projective variety $B$, and a family of abelian varieties

$$
\mathcal{A} \rightarrow B
$$

parametrized by $B$, together with a dominating rational map $\phi: \mathcal{A} \rightarrow \rightarrow$ $X$ which is non-constant on the fibers of $\pi$. We then apply Lemma 1.42 to conclude that $\widetilde{\Psi}_{X}=0$ on a Zariski open set of $X$.

EXAMPLE 2.14. A uniruled variety is a variety $X$ which admits a dominating generically finite rational map $\phi: Y \times \mathbb{P}^{1} \rightarrow X$. Uniruled varieties satisfy Lang's conjecture since there is a surjective holomorphic map $E \rightarrow \mathbb{P}^{1}$, for any elliptic curve $E$.

ExAMPLE 2.15. Abelian varieties or varieties birationally equivalent to them obviously satisfy Lang's conjecture.

EXAMPLE 2.16. Algebraic $K 3$ surfaces and there punctual Hilbert schemes satisfy Lang's conjecture since they are swept out by elliptic curves. (see Lemma 1.51.

Example 2.17. Smooth double covers $r: Y \rightarrow \mathbb{P}^{n}$ of the projective space which are not of general type satisfy Lang's conjecture. Indeed, let $\Sigma \subset \mathbb{P}^{n}$ be the branch locus. It has to be a smooth hypersurface of degree $2 d$, with $d \leq n+1$ for the canonical bundle of $Y$ not to be ample. Consider lines in $\mathbb{P}^{n}$ which are tangent to $\Sigma$ at $d-2$ points. They are parameterized by a family of dimension $\geq 2 n-2-(d-2) \geq n-1$. One can show by intersection theory that these lines sweep out $\mathbb{P}^{n}$. One can also show that the generic such line $l$ meets $\Sigma$ with simple tangency at $d-2$ points, and transversally at the 4 remaining points. It follows immediately that the curve $r^{-1}(l)$ has a normalization which is an elliptic curve, so that $Y$ is swept out by elliptic curves. Note that these varieties admit in fact presumably infinitely many coverings by elliptic curves. Indeed, replace in the above reasoning, lines by rational curves of degree $l$. They have $(n+1)(l+1)-4$ parameters and they meet $\Sigma$ in $2 d l$ points. Those which meet $\Sigma$ tangentially along $d l-2$ points and simply along 4 points form a family of expected dimension $(n+1)(l+1)-4-(d l-2) \geq n-1$, for $d \leq n+1$. Hence they should cover $\mathbb{P}^{n}$, and a supplementary argument involving intersection theory should show that they indeed cover it.

EXAMPle 2.18. Let $X$ be a smooth cubic hypersurface in $\mathbb{P}^{5}$, and let $F \subset \operatorname{Grass}(2,6)$ be the Fano variety of lines contained in $X$. It is 
known $[2]$ that $F$ is a symplectic holomorphic variety, that is, it carries a nowhere degenerate holomorphic 2-form, which implies in particular that its canonical bundle is trivial. We claim that $F$ is swept out by surfaces birationally isomorphic to abelian surfaces. Indeed, if $Y \subset X$ is a hyperplane section, the Fano surface $\Sigma_{Y}$ of lines in $Y$ is contained in $F$. When $Y$ acquires a singular point, this Fano surface becomes birationally equivalent to the second symmetric product of the curve $C$ of lines passing through the singular point $y$, and $C$ is a complete intersection of a cubic and a quadric in $\mathbb{P}^{3}$ hence has genus 4 (cf $[\mathbf{1 5}]$ ). Indeed, if $l \subset Y$ is a line in $Y$ which does not pass through $y$, the plane $P:=<l, y>$ intersects $Y$ along a cubic curve, which has $l$ as a degree 1 irreducible component, and which is singular at $y$. Such a curve is necessarily the union of three lines, two of them passing through $y$.

Next, if we impose two more singular points to $Y$, we find that the curve $C$ acquires two nodes, so that its genus decreases to 2 . Hence the surface $\Sigma_{Y}$ becomes birationally equivalent to the second symmetric product of a genus 2 curve, hence to an abelian surface. Finally there is a two dimensional family of hyperplane sections of $X$ having three nodes, and it is easy to check that the corresponding $\Sigma_{Y}$ cover $F$.

We considered here mostly Calabi-Yau (i.e. $K$-trivial) examples and this for the following reason. It is conjectured and proved in dimension $3[\mathbf{5 2}]$ that a smooth projective variety which satisfies $h^{0}\left(X, K_{X}^{\otimes r}\right)=$ $0, \forall r>0$, is uniruled. Hence, by example 2.14, such varieties satisfy Lang's conjecture. On the other hand, if $X$ does not satisfy the condition above, it admits a fibration, given by the sufficiently divisible pluricanonical systems, whose generic fiber can be shown to be of Kodaira dimension 0 i.e $h^{0}\left(X, K_{X}^{\otimes r}\right) \leq 1, \forall r>0$, with equality for sufficiently divisible $r$, and is positive dimensional if and only if $X$ is not of general type ( $\mathrm{cf}[\mathbf{5 3}]$ ). Finally, varieties of Kodaira dimension 0 are conjectured to be birationally equivalent to finite quotient of singular $K$-trivial varieties (this is proved in dimension $3[39]$ ). All this put together suggests that a variety which is not of general type is either uniruled, or fibered with general fiber birationally equivalent to a finite quotient of a singular positive dimensional $K$-trivial variety. This would allow to restrict Lang's conjecture to the $K$-trivial case.

2.1.3. Algebraic measure-hyperbolicity . Let us start with the following observation :

Lemma 2.19. If $X$ is a variety of general type, and $L$ is an ample line bundle on $X$, there exists a constant $A>0$ such that for any 
covering family of curves on $X$, that is any diagram

$$
\begin{gathered}
\phi: \quad \mathcal{C} \rightarrow X \\
\pi \downarrow \\
B \quad,
\end{gathered}
$$

where $\phi$ is dominating, non constant on the fibers of $\pi$, and $\pi$ is projective with generic fiber a curve $C$ of genus $g$, one has

$$
2 g-2 \geq A \operatorname{deg} \phi_{C}{ }^{*} L,
$$

where $\phi_{C}:=\phi_{\mid C}$.

Proof. Since $X$ is of general type, the line bundle $K_{X}^{\otimes r} \otimes L^{-1}$ is effective for $r$ sufficiently large. Let us choose a section of this line bundle, and let $D$ be its divisor. Since the curves $C$ of the family cover $X$, they do not stay in any component of $D$ hence they satisfy $\operatorname{deg} \phi_{C}^{*} D \geq 0$. On the other hand, because $\phi$ is dominating, it is generically of maximal rank, which implies that the normal bundle $\phi_{C}{ }^{*} T_{X} / \phi_{C_{*}}\left(T_{C}\right)$ is generically generated by global sections on $C$. Hence we have

$$
\operatorname{deg} \phi_{C}{ }^{*} T_{X} / \phi_{C *}\left(T_{C}\right) \geq 0 .
$$

It follows that $\operatorname{deg} \phi_{C}{ }^{*} K_{X} \leq \operatorname{deg} K_{C}=2 g-2$. Hence

$$
\operatorname{deg} \phi_{C}{ }^{*} L \leq r \operatorname{deg} \phi_{C}{ }^{*} K_{X} \leq r(2 g-2) .
$$

The following definition is the natural counterpart of definition 2.2 :

Definition 2.20. A variety is algebraically measure-hyperbolic if it satisfies the conclusion of Lemma 2.19.

With this definition, Lemma 2.19 can be rephrased saying that a variety which is of general type is algebraically measure-hyperbolic. A variety which is not algebraically measure-hyperbolic can be swept out by curves $C$ of genus $g(C)$, with $g(C) / \operatorname{deg} C$ arbitrarily small.

On the other hand, if Lang's conjecture 2.13 is true, an $X$ which is not of general type is dominated by a family of abelian varieties, i.e., there exists a diagram

$$
\begin{array}{ccc}
\phi: & \mathcal{A} & \rightarrow X \\
\pi \downarrow & \\
B &
\end{array}
$$

where $\phi$ is rational, dominating, non constant on the fibers of $\pi$, and $\pi$ is projective with generic fiber an abelian variety. We may assume that $\pi$ has a section, so that multiplication by $n$, denoted by $n \cdot$, acts 
on the fibers of $\pi$. Hence we get $\phi_{n}:=\phi \circ n \cdot: \mathcal{A} \rightarrow X$. Now, since $n^{*}$ is multiplication by $n^{2}$ on $H^{2}\left(A_{b}, \mathbb{Z}\right)$, for each fiber $A_{b}$ of $\pi$, it follows that if $C \subset A_{b}$ is a curve which does not meet the indeterminacy locus of $\phi$ and $\phi_{n}$, we have

$$
\operatorname{deg} \phi_{n}^{*} L_{\mid C}=n^{2} \operatorname{deg} \phi^{*} L_{\mid C} .
$$

Hence, starting with one covering family for $\mathcal{A}$, and applying $\phi_{n}$ to it, we see that, letting $n$ tends to infinity, we can construct covering families by curves of fixed genus and of arbitrarily large degree.

This shows that varieties which are rationally dominated by a family of abelian varieties are not algebraically measure hyperbolic. Hence a weaker version of Lang's conjecture 2.13 would be the following converse to Lemma 2.19 :

CONJECTURE 2.21. If $X$ is not of general type, it is not algebraically measure-hyperbolic.

Definition 2.20 raises a number of questions.

Question. If $X$ is not algebraically measure-hyperbolic, is it true that it is not measure-hyperbolic? Is it true that $\Psi_{X}$ or $\widetilde{\Psi}_{X}$ vanishes on a Zariski open set?

The answer to this question would show that conjecture 2.21 implies Kobayashi's conjecture.

Question. If $X$ is not algebraically measure-hyperbolic, is it true that its Kobayashi infinitesimal pseudo-metric is degenerate almost everywhere?

None of these questions seems to have an obvious answer. Looking at the proof of Proposition 2.1, we see that if $X$ is not algebraically measure hyperbolic, it is swept out by curves $\phi_{n}: C_{n} \rightarrow X$, such that the integral of the hyperbolic Kähler form of $C$ is arbitrarily small compared with the integral over $C$ of a given Kähler form on $X$. In order to solve the second question above, we would need that this comparison also holds pointwise everywhere along $C_{n}$.

2.2. $K$-correspondences and a variant of Kobayashi's conjecture. We describe here the construction of a pseudo-volume form $\Phi_{X}$ on any complex manifold $X$, which satisfies the decreasing volume property with respect to certain multivalued maps, that we shall call $K$ correspondences. On one hand, the analogue of Griffiths-Kobayashi's 
theorem 1.29 is also true for this variant of the Kobayashi-Eisenman pseudo-volume form, and on the other hand, we can show that many Calabi-Yau varieties carry self-K-correspondences dilating their canonical volume form. Hence we can show many instances of the analogue of Kobayashi's conjecture for $\Phi_{X}$.

2.2.1. K-correspondences. If $\phi: X \rightarrow Y$ is a holomorphic map, we have the Jacobian determinant map

$$
\wedge^{n} \phi_{*}: \bigwedge^{n} T_{X} \rightarrow \phi^{*} \bigwedge^{n} T_{Y}
$$

$K$-correspondences are multivalued holomorphic maps $\phi$ from a smooth complex manifold to another one (of the same dimension), satisfying the property that there is an associated Jacobian determinant map, which is holomorphic. This is formalized as follows :

Definition 2.22. [67] A $K$-correspondence from $X$ to $Y$, where $X$ and $Y$ are smooth of the same dimension $n$, is a reduced closed analytic subset $\Sigma \subset X \times Y$ satisfying the following properties :

(1) Each irreducible component of $\Sigma$ has dimension $n$ and is generically of maximal rank over $X$ and $Y$.

(2) The restriction $\operatorname{pr}_{1 \mid \Sigma}$ is proper.

(3) Let $\tau: \widetilde{\Sigma} \rightarrow \Sigma$ be a desingularization, and let $f:=p r_{1} \circ \tau, g=$ $p r_{2} \circ \tau$. Then the ramification divisors $R_{f}$ and $R_{g}$ satisfy the inequality

$$
R_{f} \leq R_{g}
$$

The last property can be rephrased as follows : $R_{f}$ is the hypersurface defined by the vanishing of the Jacobian determinant map $\wedge^{n} f_{*}: \bigwedge^{n} T_{\widetilde{\Sigma}} \rightarrow f^{*}\left(\bigwedge^{n} T_{X}\right)$, and similarly $R_{g}$ is the hypersurface defined by the vanishing of $\wedge^{n} g_{*}: \bigwedge^{n} T_{\widetilde{\Sigma}} \rightarrow g^{*}\left(\bigwedge^{n} T_{Y}\right)$. Then condition 3 says that the map

$$
\wedge^{n} g_{*} \circ \wedge^{n} f_{*}^{-1}: f^{*}\left(\bigwedge^{n} T_{X}\right) \rightarrow g^{*}\left(\bigwedge^{n} T_{Y}\right)
$$

is holomorphic. This map will be called the generalized Jacobian determinant and denoted by $J_{\widetilde{\Sigma}}$. For easy reasons, it is in fact already defined on the normalization of $\Sigma$.

$K$-correspondences compose as follows. If $\Sigma \subset X \times Y$ and $\Sigma^{\prime} \subset Y \times$ $Z$ are $K$-correspondences, consider the fibered product $\Sigma^{\prime \prime}:=\Sigma \times_{Y} \Sigma^{\prime}$. The composed correspondence $\Sigma^{\prime} \circ \Sigma \subset X \times Z$ is defined as the union of those components of the image of $\Sigma^{\prime \prime}$ in $X \times Z$ via the natural map, which are generically of maximal rank $n$ over $X$. It is easy to show that they are also generically of maximal rank over $Z$ and have dimension 
$n$. Furthermore, properness over $X$ follows from the same property for $\Sigma$ and $\Sigma^{\prime}$. Finally, one checks that the composition in the above sense of two $K$-correspondences also satisfy property 3 , hence is also a $K$-correspondence.

We have next the corresponding notion of $K$-isocorrespondence : a $K$-isocorrespondence between $X$ and $Y$ is a $K$-correspondence $\Sigma \subset$ $X \times Y$, such that the transposed correspondence ${ }^{t} \Sigma \subset Y \times X$ is also a $K$-correspondence. In other words the following properties should be satisfied :

(1) Every component of $\Sigma$ is of dimension $n$ and generically of maximal rank over $X$ and $Y$.

(2) The restrictions to $\Sigma$ of $p r_{1}$ and $p r_{2}$ are proper.

(3) With the same notations as in Definition 2.22, we have

$$
R_{f}=R_{g} \text {. }
$$

Note that the last property means equivalently that the generalized Jacobian determinant map $J_{\widetilde{\Sigma}}$ does not vanish on $\widetilde{\Sigma}$, thus providing a canonical isomorphism between $f^{*}\left(\bigwedge^{n} T_{X}\right)$ and $g^{*}\left(\bigwedge^{n} T_{Y}\right)$, or equivalently an inverse isomorphism betweem the line bundles $f^{*} K_{X}$ and $g^{*} K_{Y}$.

ExAmple 2.23. If $\operatorname{dim} X=\operatorname{dim} Y$ and $\phi: X \rightarrow Y$ is a holomorphic map which is generically of maximal rank, then the graph of $\phi, \Gamma_{\phi} \subset$ $X \times Y$, is a $K$-correspondence. If $\phi$ is an étale and proper morphism, $\Gamma_{\phi}$ is a $K$-isocorrespondence.

ExAmple 2.24. If $A$ is an abelian variety, and $\left(m, m^{\prime}\right)$ are a pair of coprime integers, then

$$
A \stackrel{\left(m, m^{\prime}\right)}{\longrightarrow} A \times A
$$

is a $K$-isocorrespondence. These examples have the very special property that both maps $f, g$ (or $p r_{1}, p r_{2}$ in this case) are unramified. Clozel and Ullmo [18]constructed curves possessing an infinite number of self-correspondences satisfying this last property.

In contrast to the above example where the degrees of the map $f$ and $g$ are different (they are equal to $m^{2 n}$ and $m^{\prime 2 n}$ respectively), we have the following result :

LEMMA 2.25. [67] If $X$ is of general type, a K-autocorrespondence $\Sigma \subset X \times X$ satisfies

$$
\operatorname{deg} f=\operatorname{deg} g
$$

where $f, g$ are defined as in Definition 2.22. 
The following also shows that having non trivial $K$-autocorrespondences is restrictive, at least for varieties which are of general type (while the results in next section tend to show that they are very easy to construct in the $K$-trivial case.)

Lemma 2.26. [67] A general curve of genus $g \geq 3$ does not admit non trivial $K$-autocorrespondences.

The next natural example of $K$-isocorrespondences comes from the study of quotient singularities.

EXAMPLE 2.27. Let $X$ be smooth and let $G$ be a finite group acting on $X$. Assume that the isotropy groups $G_{x}$ act via $S L(n)$ on the tangent spaces $T_{X, x}$ and that there exists a crepant resolution

$$
\tau: Y \rightarrow X / G
$$

Then the graph

$$
\Gamma_{q} \subset X \times Y
$$

of the meromorphic map $q: X \rightarrow Y$ is a $K$-isocorrespondence.

2.2.2. Existence theorem for $K$-correspondences. Consider a degree 3 curve $E$ in $\mathbb{P}^{2}$. It is an elliptic curve. Multiplication by -2 is easy to construct on it by means of projective geometry. Indeed, for $x \in E$, consider the tangent line $l$ to $E$ at $x$. This line meets $E$ at another point $y$. It is easy to see that the map $x \mapsto y$ is for an adequate choice of origin, the multiplication by -2 .

It turns out that this construction generalizes in higher dimension to provide more generally interesting $K$-autocorrespondences on many Calabi-Yau manifolds.

The Calabi-Yau manifolds we will consider are the following : recall first that a rationally connected variety $Y$ is a variety satisfying the property that through any two points $x, y \in Y$ there passes at least one rational curve. This curve can then be chosen to be smooth, and to have arbitrarily ample normal bundle in $Y$ (see $[\mathbf{4 7}]$ ). We have the following natural way to construct $K$-trivial varieties : if $Y$ is smooth and $\sigma$ is a non zero section of $-K_{Y}$, such that $X:=\operatorname{div} \sigma$ is smooth, then $K_{X}$ is trivial by the adjunction formula :

$$
K_{X}=K_{Y} \otimes \mathcal{O}_{X}(X) .
$$

If furthermore $Y$ is a rationally connected variety, we shall say that $X$ is a Calabi-Yau hypersurface in a rationally connected variety, (omitting to mention the fact that we want $\left.X \in\left|-K_{Y}\right|\right)$.

Calabi-Yau hypersurfaces in rationally connected varieties are the most common Calabi-Yau varieties. All Calabi-Yau complete intersections in Fano toric varieties fall in this category. In [67], there are given 
examples of Calabi-Yau varieties which do not belong to this category. (Note that abelian varieties, or symplectic holomorphic varieties, which have trivial canonical bundle, cannot be constructed this way, but since they do not satisfy the vanishing

$$
H^{i}\left(X, \mathcal{O}_{X}\right)=0,0<i<n,
$$

they are sometimes not considered as Calabi-Yau varieties.)

The result of [67] concerning the existence of $K$-autocorrespondences is the following :

Theorem 2.28. Let $X$ be a Calabi-Yau hypersurface in a rationally connected variety. Then $X$ has $K$-autocorrespondences $\Sigma \subset X \times X$, which satisfy the property

$$
\operatorname{deg} f \neq \operatorname{deg} g
$$

where $f, g$ are as in Definition 2.22.

We explain now the relation between this theorem and volume forms on such $X$. Let

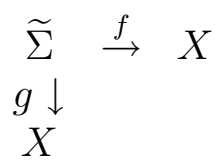

be a desingularization of $\Sigma$. Let $\eta_{X} \in H^{0}\left(X, K_{X}\right)$ be a generator. This is a holomorphic $n$-form which is everywhere non-zero on $X$. Since $\eta_{X}$ does not vanish, the ramification divisor $R_{f}$ equals the divisor of the holomorphic $n$-form $f^{*} \eta_{X}$ on $\widetilde{\Sigma}$ and similarly the ramification divisor $R_{g}$ equals the divisor of $g^{*} \eta_{X}$. The equality $R_{f}=R_{g}$ implies then, assuming that $\Sigma$ is irreducible, that for some $\lambda \in \mathbb{C}^{*}$, we have

$$
g^{*} \eta_{X}=\lambda f^{*} \eta_{X}
$$

This equality implies that

$$
g^{*}\left(\eta_{X} \wedge \overline{\eta_{X}}\right)=|\lambda|^{2} f^{*}\left(\eta_{X} \wedge \overline{\eta_{X}}\right) .
$$

Integrating over $\widetilde{\Sigma}$, and noticing that $\eta_{X} \wedge \overline{\eta_{X}}$ is proportional to a volume form on $X$, hence has a non-zero integral, we get :

$$
\operatorname{deg} g=|\lambda|^{2} \operatorname{deg} f \text {. }
$$

Now, the condition $\operatorname{deg} f \neq \operatorname{deg} g$ (inverting the role of $f$ and $g$ we may of course suppose that $\operatorname{deg} f<\operatorname{deg} g$ ), means that $|\lambda|^{2}>1$, and formula (2.18) then says that $\Sigma$ dilates the holomorphic volume form of $X$. 
REMARK 2.29. We can compose $\Sigma$ with itself $r$ times, getting a $K$-autocorrespondence $\Sigma^{\circ}$, which will satisfy the condition that the corresponding coefficient $\lambda_{r}$ is equal to $\lambda^{r}$. Since $|\lambda|>1$, this coefficient can be made arbitrarily large.

REMARK 2.30. The proof of Theorem 2.28 will show that we can construct such $\Sigma$ passing through an arbitrary pair $(x, y) \in X \times X$. In particular we may assume it passes through $(x, x)$, with arbitrary $x \in X$. Equation (2.18) then says that the generalized Jacobian determinant of $\widetilde{\Sigma}$ at any point $\sigma_{x}$ of $\widetilde{\Sigma}$ over $(x, x)$ can be made arbitrarily large.

Sketch of Proof of Theorem 2.28. The construction which is described below will give $K$-autocorrespondences with corresponding coefficient $\lambda=-2$.

Let $x, y \in X, x \neq y$. Since $Y$ is rationally connected, there is a smooth rational curve $C$ in $Y$, which has ample normal bundle in $Y$, and which intersects $X$ with a simple tangency at $x$, and transversally at $y$. Write the intersection divisor of $C$ and $X$ as

$$
C \cap X=2 x+y+z,
$$

where the zero-cycle $z$ is supported away from $x, y$ and may be assumed to be made of $C \cdot X-3$ distinct points. Choose an hypersurface $Z \subset X$ containing $z$. Consider

$$
\Sigma^{\prime}:=\left\{\left(x^{\prime}, y^{\prime}, C^{\prime}\right), C^{\prime} \cap X=2 x^{\prime}+y^{\prime}+z^{\prime}\right\},
$$

where $C^{\prime}$ is a deformation of $C, x^{\prime}, y^{\prime} \in X$, and $z^{\prime}$ is subject to the condition $z^{\prime} \subset Z$.

Our $K$-correspondence will be defined as the image of $\Sigma^{\prime}$ in $X \times X$ via the map

$$
\sigma=\left(C^{\prime}, x^{\prime}, y^{\prime}\right) \mapsto\left(x^{\prime}, y^{\prime}\right) .
$$

We first claim that for a generic choice of $Z, \operatorname{dim} \Sigma^{\prime}=\operatorname{dim} \Sigma=n$. Indeed, the space of deformations of $C$ in $Y$ has dimension

$$
h^{0}\left(C, N_{C / Y}\right)=-K_{Y} \cdot C+n-2,
$$

(cf [46]), and the number of conditions imposed to $C^{\prime}$ is $X \cdot C-2$, since we want $C^{\prime}$ to remain tangent to $X$ at $x^{\prime}$, and $z^{\prime}$ to stay in $Z$. Since $X \in\left|-K_{Y}\right|$, we find that the expected dimension of $\Sigma^{\prime}$ is $n$. An easy argument shows that this dimension count is correct for an adequate choice of $Z$. The proof that $\Sigma$ is a $K$-isocorrespondence with coefficient $\lambda=-2$ follows now from Proposition 2.31 below. 
Proposition 2.31. Notations being as above, we have

$$
2 p r_{1}^{*} \eta_{X}+p r_{2}^{*} \eta_{X}=0
$$

on $\Sigma$.

This proposition is an application of Mumford's theorem [54] on pull-backs of holomorphic forms via correspondences, or more precisely its higher dimensional generalization (see eg [69], III.10) :

TheOrem 2.32. Let $\Gamma \subset Y \times X$ be an algebraic subset, which is generically finite over $Y$. Assume there exists a proper algebraic subset $X^{\prime} \subset X$, such that for any $y \in Y$, the 0 -cycle $\Gamma_{*}(y)$ is rationally equivalent to a 0 -cycle supported on $X^{\prime}$; then for any top degree holomorphic form $\eta_{X}$ on $X$, we have

$$
\Gamma^{*} \eta_{X}=0
$$

on $Y$.

Indeed, we apply Mumford's theorem to the correspondence $\Gamma$ between $\widetilde{\Sigma}$ and $X$, which to $\sigma=\left(x^{\prime}, y^{\prime}, C^{\prime}\right)$ associates the 0 -cycle

$$
\Gamma_{*}(\sigma):=2 x^{\prime}+y^{\prime} \in \mathcal{Z}_{0}(X)
$$

The 0 -cycle $2 x^{\prime}+y^{\prime}$ is equal to $C^{\prime} \cdot X-z^{\prime}$. By assumption $z^{\prime}$ is supported on $Z$. On the other hand, since $Y$ is rationally connected, one can show that its Chow group $\mathrm{CH}_{1}$ of 1 -cycles "has the size" of the Chow group of 0 -cycles of a $n$-1-dimensional variety. Hence it follows that there exists a $Z^{\prime} \subset X$ such that 0 -cycles of the form $C^{\prime} \cdot X$ are supported on $Z^{\prime}$ modulo rational equivalence. Now Mumford's Theorem says that in this situation, the pull-back $\Gamma^{*} \eta_{X}$ vanishes as a $n$-form on $\widetilde{\Sigma}$. But this pull-back is obviously equal to $2 p r_{1}^{*} \eta_{X}+p r_{2}^{*} \eta_{X}$.

2.2.3. A variant of the Kobayashi-Eisenman pseudo-volume form. The fact that for a $K$-correspondence, we have a holomorphic generalized Jacobian determinant map, together with the fact that $K$ correspondences can be composed, and thus can be considered as generalized morphisms, suggest the following variant $\Phi_{X}$ of the KobayashiEisenman pseudo-volume form $\Psi_{X}$, where one replaces holomorphic maps from $D^{n}$ to $X$ with $K$-correspondences from $D^{n}$ to $X$.

Definition 2.33. For $x \in X, \zeta \in \bigwedge^{n} T_{X, x}$, the Hermitian pseudonorm of $\zeta$ with respect to $\Phi_{X}$ is defined as

$$
\Phi_{X, x}(\zeta)=\frac{1}{\lambda}
$$


where $\lambda=\operatorname{Sup}_{\Sigma, \sigma}\left\{|\mu|, J_{\widetilde{\Sigma}}\left(\frac{\partial}{\partial z_{1}} \wedge \ldots \frac{\partial}{\partial z_{n}}\right)=\mu \zeta\right\}$. Here $\Sigma$ runs over all $K$-correspondences from $D^{n}$ to $X$ and $\sigma$ over all points of $\widetilde{\Sigma}$ which are over $(0, x)$.

The first properties of $\Phi_{X}$ are the following : first of all, one obviously has

$$
\Phi_{X} \leq \Psi_{X}
$$

Next, $\Phi_{X}$ satisfies the decreasing volume property with respect to $K$ correspondences:

LEMma 2.34. If $\Sigma \subset X \times Y$ is a $K$-correspondence, then, with the same notations as above,

$$
g^{*} \Phi_{Y} \leq f^{*} \Phi_{X}
$$

as pseudo-volume forms on $\widetilde{\Sigma}$. In particular, if $\Sigma$ is a $K$-isocorrespondence, this inequality becomes an equality.

Less obvious are the following results, whose proof follows the same lines as those of Theorems 1.26 and 1.29 :

TheOrem 2.35. [67] If $X=D^{n}$ or $X$ is a product of curves of genus $\geq 2, \Phi_{X}=\Psi_{X}$, that is $\Phi_{X}=\kappa_{n}$ in the first case, and (up to a coefficient) the product of the hyperbolic volume forms on each factor in the second case.

THEOREM 2.36. [67] If $X$ is projective of general type, then $\Phi_{X}>0$ on a non-empty Zariski open set of $X$. (The proof even shows that the lower bound (1.11) for $\Psi_{X}$ is also valid for $\Phi_{X}$.)

The important point in the proof of these statements is the following. One has in both cases to study $K$-correspondences $\Sigma$ from $D^{n}$ to $X$, where $X$ will be either $D^{n}$, or a product of curves, or a variety of general type. Let $\mu$ be a pseudo-volume form on $X$ which satisfies the conditions :

$$
\begin{array}{r}
i \partial \bar{\partial} \log \mu>0, \\
(i \partial \bar{\partial} \log \mu)^{n} \geq c_{n} \mu,
\end{array}
$$

where $c_{n}$ is as in section (1.2.1). Then the function

$$
g^{*} \mu / f^{*} \kappa_{n}
$$

is $C^{\infty}$ on $\widetilde{\Sigma}$, because we have the condition

$$
R_{f} \leq R_{g}
$$

which will imply that the pseudo-volume form on the numerator vanishes more than the one on the denominator. The proof of the theorem 
is then, as in Ahlfors-Schwarz Lemma, an application of the maximum principle to this function on $\widetilde{\Sigma}$. Here the properness of $\widetilde{\Sigma}$ over $D^{n}$ is used in an essential way to produce a maximum.

To conclude, Theorem 2.28 gives the following :

TheOREM 2.37. Let $X$ be a variety which admits a fibration :

$$
\phi: X \rightarrow B
$$

whose generic fiber is a Calabi-Yau hypersurface in some rationally connected variety. Then $\Phi_{X}=0$ on a Zariski open set of $X$.

The proof is elementary. One first observes that the construction which gives the proof of Theorem 2.28 can be made in family over a Zariski open set $U$ of $B$, which provides a $K$-autocorrespondence

$$
\Sigma_{U} \subset X_{U} \times X_{U},
$$

where $X_{U}=\phi^{-1}(U)$. Let $\mu$ be a volume form on $B$, and consider the volume form

$$
\Omega:=\phi^{*} \mu \wedge \Omega_{r e l, c a n},
$$

where $\Omega_{\text {rel,can }}$ is the relative canonical volume form, which is locally over $U$ equal to $\phi^{*} \alpha \eta \wedge \bar{\eta}$ for some function $\alpha$ on $U$, where $\eta$ is a section of the relative canonical bundle, and where $\alpha$ is determined by the condition that this relative volume form has integral 1 on the fibres of $\phi$.

Then formula (2.18) along the fibres of $\phi$, (where one notes that for topological reasons $\lambda$ is constant), implies that if

$$
\begin{aligned}
& \widetilde{\Sigma_{U}} \stackrel{f}{\rightarrow} X_{U} \\
& g \downarrow \\
& X_{U}
\end{aligned}
$$

is a desingularization of $\Sigma_{U}$, then

$$
g^{*} \Omega=|\lambda|^{2} f^{*} \Omega \text {. }
$$

On the other hand, we have by Lemma 2.34 :

$$
g^{*} \Phi_{X_{U}}=f^{*} \Phi_{X_{U}} .
$$

This immediately implies that $\Phi_{X_{U}}=0$. Indeed, writing $\Phi_{X_{U}}=\gamma \Omega$, for some uppersemicontinuous function $\gamma$ on $X_{U}$, these two relations give

$$
g^{*} \gamma=|\lambda|^{2} f^{*} \gamma
$$

Since $\gamma$ has a maximum on each fiber of the map $\widetilde{\Sigma}_{U} \rightarrow U$, this last relation, together with the fact that $|\lambda|^{2}>1$, implies that $\gamma=0$. 
In conclusion, we have shown that the modified pseudo-volume form $\Phi_{X}$ satisfies the analogue of the Griffiths-Kobayashi-Ochiai Theorem 1.29 , and equals $\Phi_{X}$ for the polydisk or more generally products of curves. On the other hand, Theorem 2.37 shows that $\Phi_{X}=0$ for a reasonably general class of varieties which are not of general type, thus proving in many instances the analogue of Kobayashi's conjecture 0.1 for $\Phi_{X}$.

Thus the natural question we are led to is the following :

Question. How do $\Phi_{X}, \widetilde{\Psi}_{X}$ and $\Psi_{X}$ compare? (Note that we always have $\Phi_{X} \leq \Psi_{X}, \widetilde{\Psi}_{X} \leq \Psi_{X}$.) Are $\Phi_{X}$ and $\widetilde{\Psi}_{X}$ equivalent? There is a variant $\widetilde{\Phi}_{X}$ of $\Phi_{X}$ which is bimeromorphically invariant (cf $[67])$. Are $\widetilde{\Phi}_{X}$ and $\widetilde{\Psi}_{X}$ equivalent?

\section{General hypersurfaces in projective space}

\subsection{Hyperbolicity of hypersurfaces and their complements.} We consider general hypersurfaces of degree $d$ in projective space $\mathbb{P}^{n+1}$. For small degree $d$, it is easy to show that neither the hypersurfaces, nor their complement are algebraically hyperbolic. We describe here methods which allow to prove algebraic hyperbolicity for the general hypersurface when the degree becomes large enough (an explicit, optimal bound will be given.) We also give an argument for the algebraic hyperbolicity of the complement. We conclude with a sketch of the methods used to prove the analogous (but much more difficult) statements for Kobayashi hyperbolicity.

3.1.1. Algebraic hyperbolicity. Algebraic hyperbolicity of general hypersurfaces of degree $d \geq 2 n+2$ in $\mathbb{P}^{n+1}$ has been established by Clemens [14].

TheOREM 3.1. (Clemens) If $d \geq 2 n+1$, a general hypersurface degree $d$ in $\mathbb{P}^{n+1}$ does not contain any rational curve. More generally, there is the following lower bound for the genus $g$ of curves $C$ contained in a general hypersurfaces of degree $d$ in $\mathbb{P}^{n+1}$ :

$$
2 g-2 \geq(d-2 n-1) \operatorname{deg} C .
$$

REMARK 3.2. One can check that any hypersurface of degree $d \leq$ $2 n-1$ in $\mathbb{P}^{n+1}$ contains lines, which shows that the theorem above is not far from being optimal.

Ein [26] subsequently generalized such estimates to subvarieties of any codimension. His result is the following : 
TheOREM 3.3. (Ein) Fix an integer $k \leq n-1$ (which will be the codimension). Then for $d \geq n+k+2$ and $X$ a general hypersurface of degree $d$ in $\mathbb{P}^{n+1}$, any codimension $k$ subvariety $Y \subset X$ has effective canonical bundle (meaning that any desingularization of $Y$ satisfies this property). More precisely, for any desingularization $\widetilde{Y} \rightarrow Y$ of $Y$, the line bundle

$$
K_{\widetilde{Y}}(-d+n+k+2)
$$

is effective.

Clemens theorem is recovered as the case $k=n-1$ of this theorem.

Finally, $\mathrm{Xu}[\mathbf{7 2}]$ got better results in the case of subvarieties of codimension 1, which can be explained by the fact that a codimension 1 subvariety of a generic hypersurface of dimension $\geq 2$ in projective space is cut out by a hypersurface in ambiant space.

In [65], we presented the proof of theorems 3.1 and 3.3 in the following way, which led to further improvements $([\mathbf{6 5}],[\mathbf{5 7}],[\mathbf{1 6}])$ which we will describe later on.

Proof of Theorem 3.3. The idea is as follows : let $U \subset H^{0}\left(\mathbb{P}^{n+1}, \mathcal{O}_{\mathbb{P}^{n+1}}(d)\right)$ be the Zariski open set parameterizing smooth hypersurfaces. Let

$$
\pi: \mathcal{X} \rightarrow U, \mathcal{X} \subset \mathbb{P}^{n+1} \times U
$$

be the universal family. Assume that for general $X$ there is given a subvariety $Y \subset X$ of codimension $k$. As there are only countably many components of the relative Hilbert scheme parameterizing subvarieties of codimension $k$ in fibers of $\pi$, a Baire argument shows that there exist a quasi-projective variety $B$, a dominant morphism $\phi: B \rightarrow U$, and a relative subvariety of codimension $k$

$$
\mathcal{Y} \subset \mathcal{X}_{B}
$$

where $\mathcal{X}_{B}:=\mathcal{X} \times_{U} B$, such that $Y \subset X$ is one fiber $Y_{b} \subset X_{b}$ for some $b \in B$. Note that we may assume that $B$ is smooth and $\phi$ is étale, by restricting to a locally closed subset of $B$. It follows that the bundle $\phi^{*} \Omega_{\mathcal{X}}$ is isomorphic to $\Omega_{\mathcal{X}_{B}}$, where we denote also by $\phi: \mathcal{X}_{B} \rightarrow \mathcal{X}$ the induced map. We shall denote by $\widetilde{\mathcal{Y}}$ a desingularization of $\mathcal{Y}$. It induces a desingularization of the generic fiber $Y_{b}$.

The strategy to produce sections of $K_{\widetilde{Y}_{b}}(-d+n+k+2)$ is now the following. Let $N:=\operatorname{dim} B$. Then

$$
\operatorname{dim} \widetilde{\mathcal{Y}}=N+n-k
$$


and

$$
K_{\tilde{\mathcal{Y}}}=\bigwedge^{N+n-k} \Omega_{\tilde{\mathcal{Y}}}
$$

Furthermore, for generic $b$, the adjunction formula shows that

$$
K_{\widetilde{\mathcal{Y}} \widetilde{Y}_{b}}=K_{\widetilde{Y_{b}}} .
$$

Hence, to construct non-zero sections of $K_{\widetilde{Y}_{b}}(-d+n+k+2)$, it will suffice to prove:

LEMMA 3.4. The bundle

$$
\bigwedge^{N+n-k} \Omega_{\mathcal{X}_{\mid X_{b}}}(-d+n+k+2) \cong \bigwedge^{N+n-k} \Omega_{\widetilde{\mathcal{X}}_{B_{\mid X}}}(-d+n+k+2)
$$

is generated by global sections.

Indeed, restricting these sections to $Y_{b} \subset X_{b}$ and projecting them further to $\bigwedge^{N+n-k} \Omega_{\widetilde{\mathcal{Y}}_{\mid Y_{b}}}$ will produce via the identifications (3.21) and (3.22), the desired non-zero section of $K_{\widetilde{Y}_{b}}(-d+n+k+2)$.

Proof of Lemma 3.4. Since we have

$$
\operatorname{rank} \Omega_{\mathcal{X} \mid X_{b}}=N+n, \operatorname{det} \Omega_{\mathcal{X} \mid X_{b}}=K_{X_{b}}=\mathcal{O}_{X_{b}}(d-n-2),
$$

there is a canonical isomorphism :

$$
\bigwedge^{N+n-k} \Omega_{\mathcal{X}}(-d+n+k+2)_{\mid X_{b}} \cong \bigwedge^{k} T_{\mathcal{X}}(k)_{\mid X_{b}} .
$$

Lemma 3.4 follows then from the following

LEMma 3.5. The bundle $T_{\mathcal{X}}(1)_{\mid X_{b}}$ is generated by global sections.

As mentioned in remark 3.2, these results are not far from being optimal. However Clemens conjectured that they could be improved by 1 , which was shown in $[66]$.

THEOREM 3.6. (Voisin) If $d \geq 2 n$, and $n \geq 3$, a general hypersurface $X$ of degree $d$ in $\mathbb{P}^{n+1}$ contains no rational curve.

If $d \geq 2 n+1$, and $C \subset X$ is any curve, one has

$$
h^{0}\left(\widetilde{C}, K_{\widetilde{C}}(-1)\right) \neq 0,
$$

which provides the following lower bound for the genus of $C$ :

$$
2 g-2 \geq \operatorname{deg} C \text {. }
$$


More generally, for any $2 \leq k \leq n-1$, if $d \geq n+k+1$, and $X$ is general of degree $d$, any codimension $k$ subvariety of $X$ has effective canonical bundle.

This theorem is now optimal, in view of remark 3.2. One can now go further and try to see what are the counterexamples to the first statement when the degree goes smaller. Pacienza [57] shows the following :

Theorem 3.7. (Pacienza) Assume $n \geq 4$. Then the only rational curves contained in a general hypersurface of degree $d=2 n-1$, are the lines contained in $X$.

More generally, if $d=n+k, k \geq 4$, and $X$ is general of degree $d$, the only codimension $k$ subvariety of $X$ which does not have effective canonical bundle is the variety swept-out by lines.

This result has been further generalized by Clemens and Ran [16], who study more generally subvarieties $Y$ in generic hypersurfaces $X$ of degree $d$ in projective space $\mathbb{P}^{n+1}$, satisfying the condition

$$
H^{0}\left(\widetilde{Y}, K_{\widetilde{Y}}(t)\right)=0,
$$

where $t$ is a non negative integer.

THEOREM 3.8. Let $X$ be general and $Y \subset X$ be a subvariety of codimension $k$ with desingularization $\tilde{Y}$. Assume that

$$
d(d+1) / 2 \geq 2 n+2+k,
$$

and let

$$
t=\operatorname{Max}\left(0,-d+n+2+\left[\frac{k+1}{2}\right]\right) .
$$

Then either $h^{0}\left(\widetilde{Y}, K_{\widetilde{Y}}(t)\right) \neq 0$, or $Y$ is ruled by lines in $X$.

Note that even for $t=0$, that is in the range

$$
d \geq n+2+\left[\frac{k+1}{2}\right]
$$

this improves Pacienza's result. Note also that if $2 d+1 \leq 3 n+2$, the generic hypersurface of degree $d$ in $\mathbb{P}^{n+1}$ contains conics (degree 2 plane curves), so that the theorem above for $k=n-1$ is sharp.

The proof of Theorem 3.6, which has been applied and refined by Pacienza and subsequently by Clemens and Ran to get Theorems 3.7 and 3.8, follows the same lines as the one described above. The only difference is that we try now to improve the statement (see the proof of Lemma 3.4) that $\bigwedge^{k} T_{\mathcal{X}}(k)_{\mid X_{b}}$ is generated by global sections, by looking at sections of $\bigwedge^{k} T_{\mathcal{X}}(k-1)_{\mid X_{b}}$. To do this, we look at sections of 
$\bigwedge^{2} T_{\mathcal{X}}(k-1)_{\mid X_{b}}$ and take their wedge product with sections of $T_{\mathcal{X}}(1)_{\mid X_{b}}$. In [65], it was mistakenly claimed that sections of $\bigwedge^{2} T_{\mathcal{X}}(1)_{\mid X_{b}}$ have no base-locus on the Grassmannian of codimension 2 subspaces of $T_{\mathcal{X} \mid X_{b}}$, which was enough, by taking wedge product with sections of $T_{\mathcal{X}}(1)_{\mid X_{b}}$, and using the isomorphism

$$
\bigwedge^{N+n-k} \Omega_{\mathcal{X}}(-d+2 n)_{\mid X_{b}} \cong \bigwedge^{n-1} T_{\mathcal{X}}(n-2)_{\mid X_{b}},
$$

to produce sections of $\bigwedge^{N+n-k} \Omega_{\widetilde{\mathcal{X}}_{B \mid X_{b}}}(-d+2 n)$ not vanishing in

$$
\bigwedge^{N+n-k} \Omega_{\tilde{\mathcal{Y}}_{\mid Y_{b}}}(-d+2 n)
$$

This was corrected in $[\mathbf{6 6}]$ : there is indeed a non-trivial base-locus but it can be described explicitly. There is correspondingly a description of the base-locus, in the Grassmannian of codimension $k$ subspaces of $T_{\mathcal{X} \mid Y_{b}}$, of the space of global sections of $\bigwedge^{k} T_{\mathcal{X}}(k-1)_{\mid X_{b}}$, for any $k \geq 2$.

The corrected proof then works as follows : By Ein's Theorem, we have only to consider the case $d=n+k+1, k \geq 2$; assume there is a family of codimension $k$ subvarieties $\mathcal{Y} \subset \mathcal{X}_{B}$, where as in the previous proof $B \rightarrow U$ is étale, and such that $Y_{b}$ does not have an effective canonical bundle. Then reasoning as in the previous proof, we see that any section of $\bigwedge^{k} T_{\mathcal{X}}(k-1)_{\mid X_{b}} \cong \bigwedge^{N+n-k} \Omega_{\mathcal{X}_{\mid X_{b}}}$ vanishes under restriction in

$$
\bigwedge^{N+n-k} \Omega_{\mathcal{Y}_{\mid Y_{b}}},
$$

for generic $b$. This means that at any point $y$ of $Y_{b}$, the tangent space

$$
T_{\mathcal{Y}, y} \subset T_{\mathcal{X}_{B}, y}
$$

has to be in the base-locus introduced above, which is a differential equation imposed to $\mathcal{Y}$. Analyzing the geometry of this differential system, we are led to the conclusion that $Y_{b}$ has to be a component of the algebraic subset of $X_{b}$ consisting of points $x \in X_{b}$ such that there is a line osculating $X_{b}$ at $x$ to order $d$. We then find a contradiction by proving that for generic $b$, this subset is irreducible of codimension $k$ and has a natural desingularization which has an effective canonical bundle.

These results suggest the following :

Conjecture 3.9. For a general hypersurface $X$ of degree $d \geq n+3$ in $\mathbb{P}^{n+1}$, the rational curves $C \subset X$ have bounded degree. 
We shall explain in section 3.2 that the assumption is necessary. Let us show the heuristic motivation for this conjecture : the condition on $d$ exactly means that the canonical bundle of $X$ is ample. Let $C \hookrightarrow X$ be a curve, with normalization $j: \widetilde{C} \rightarrow X$. Assume that $\widetilde{C}$ is rational, and assume for simplicity that it is immersed via $j$ in $X$. The adjunction formula gives $-2=\operatorname{deg} j^{*} K_{X}+\operatorname{deg} N_{\widetilde{C} / X}$. Riemann-Roch formula then says that

$$
\chi\left(N_{\widetilde{C} / X}\right)=-2-\operatorname{deg} j^{*} K_{X}+n-1 .
$$

Since $K_{X}$ is ample, we see that this number is negative when the degree of $C$ is large enough, so that the space $H^{1}\left(N_{\widetilde{C} / X}\right)$ is non trivial. Hence the space of potential obstructions to the deformations of the map $j$ (cf $[\mathbf{4 6}]$ ) is non trivial, which suggests that there are deformations of $X$ on which $C$ does not survive.

3.1.2. Geometry of the complement. Zaidenberg observed in [77] that the complement of a general hypersurface $X$ of degree $\leq 2 n+2$ in $\mathbb{P}^{n+1}$ is not hyperbolic. The reason is that there are lines in $\mathbb{P}^{n}$ which meet $X$ exactly in two points (with multiplicities). Since $\mathbb{P}^{1} \backslash$ 2 points is not hyperbolic, the result follows. We shall explain now an argument to study algebraic hyperbolicity of the complement of a general hypersurface in projective space, which is measured using only algebraically degenerate entire curves in $\mathbb{P}^{n+1} \backslash X$. Here we note that if there is a holomorphic map from $\mathbb{C}$ to $V=\mathbb{P}^{n+1} \backslash X$ whose image is contained in an algebraic curve $C^{\prime}$, then the closure $C$ of $C^{\prime}$ is rational and meets $X$ in at most 2 points.

The existence or non-existence of such curve $C$ can be done by studying the algebraic hyperbolicity of the degree $d$ cyclic cover of $\mathbb{P}^{n+1}$ ramified over $X$, which is a degree $d$ hypersurface in $\mathbb{P}^{n+2}$. Here, although this hypersurface is not generic, we may hope to apply the method explained in the previous section. What we want to study is now slightly different : find the minimum number of points of intersection of any curve $C$ with $X$. This problem was considered by $\mathrm{Xu}[\mathbf{7 3}]$ for curves in $\mathbb{P}^{2}$.

The following was proved implicitly in [65].

TheOREM 3.10. Let $X \subset \mathbb{P}^{n+1}$ be general of degree $d=2 n+r, r \geq$ 3. Then any curve $C$ meets $X$ in at least $r$ points.

Proof of Theorem 3.10. We use the following variant of Mumford's theorem 2.32 , which allows the varieties to vary with parameters. This variant was proved in [70].

THEOREM 3.11. Let $\mathcal{X} \stackrel{\pi}{\rightarrow} B$ be a smooth projective morphism, and let $\sigma_{i}, i=1, \ldots, m$ be sections of $\pi$. Choose integers $n_{i}, i=1, \ldots, m$. 
Assume that for any $b \in B$, the 0 -cycle $\sum_{i} n_{i} \sigma_{i}(b)$ is rationally equivalent to 0 in $X_{b}$. Then, for generic $b \in B$, for any $N$ and any $\alpha \in H^{0}\left(\Omega_{\mathcal{X} \mid X_{b}}^{N}\right)$, the pull-back

$$
\sum_{i} n_{i} \sigma_{i}^{*} \alpha
$$

vanishes in $\Omega_{B, b}^{N}$.

We shall apply this theorem to the following situation $: \phi: B \rightarrow U$ will be an étale map, where $U$ is the Zariski open subset of $H^{0}\left(\mathcal{O}_{\mathbb{P}^{n+1}}(d)\right)$ parameterizing smooth hypersurfaces. We assume that there is a family of curves

$$
\underset{\substack{\mathcal{C} \\ B}}{\mathcal{L}} \subset B \times \mathbb{P}^{n+1}
$$

and sections $\sigma_{i}, i=1, \ldots, m$ of $g$ such that as a 0 -cycle on $X_{b}$, we have

$$
C_{b} \cap X_{b}=\sum_{i} n_{i} \sigma_{i}(b)
$$

The contents of Theorem 3.10 is that we then have $m \geq r=d-2 n$.

Choose a line $l_{b}$ varying algebraically with $b$, such that $l_{b}$ passes through $\sigma_{1}(b)$. Since $C_{b}$ is rationally equivalent in $\mathbb{P}^{n+1}$ to $\operatorname{deg} C_{b} \cdot l_{b}$, it follows that $\sum_{i} n_{i} \sigma_{i}(b)$ is rationally equivalent in $X_{b}$ to $\operatorname{deg} C_{b} \cdot\left(l_{b} \cap X_{b}\right)$. We can thus apply Theorem 3.11 to the 0 -cycle $\sum_{i} n_{i} \sigma_{i}(b)-\operatorname{deg} C_{b}$. $\left(l_{b} \cap X\right)$. Let $N=\operatorname{dim} B$. As a consequence of Lemma 3.4 for $k=n$, we get that

$$
\bigwedge^{N} \Omega_{\mathcal{X}_{B}}(-d+2 n+2)_{\mid X_{b}}=\bigwedge^{N} \Omega_{\mathcal{X}_{B}}(2-r)_{\mid X_{b}}
$$

is generated by global sections. Now since the line $l_{b}$ is supported on an hyperplane section, it follows that the bundle

$$
\bigwedge^{N} \Omega_{\mathcal{X}_{B}}(3-r)_{\mid X_{b}} \otimes \mathcal{I}_{l_{b}}
$$

is generated by global sections. Now, if $m \leq r-1$, sections of $\mathcal{O}_{\mathbb{P}^{n+1}}(r-$ $3)$ separate the $m-1$ points $\sigma_{2}(b), \ldots, \sigma_{m}(b)$. Hence it follows that there exist sections $\alpha$ of $\bigwedge^{N} \Omega_{\mathcal{X}_{B} \mid X_{b}}$ which take arbitrary values at $\sigma_{2}(b), \ldots, \sigma_{m}(b)$ and which vanish along $l_{b}$. This contradicts the fact that for such $\alpha$, we have $\sum_{i} n_{i} \sigma_{i}^{*} \alpha=0$ in $\Omega_{B, b}^{N}$, according to Theorem 3.11 . 
3.1.3. Kobayashi's hyperbolicity . Let us first explain constructions due to Green, Zaidenberg, Ciliberto and Zaidenberg, which prove the existence of hyperbolic hypersurfaces, or of hypersurfaces with hyperbolic complements. Brody and Green [6] constructed explicit examples of hyperbolic surfaces of high degree in projective 3-space. As for the hyperbolicity of the complements, Green [34] proves the following generalization of Picard's theorem:

TheOREM 3.12. (Green) The complement of the union of $d \geq 2 n+1$ hyperplanes in general position in $\mathbb{P}^{n}$ is hyperbolically embedded.

Here for an open subset $U \subset X$, with $X$ compact, the condition that $U$ is hyperbolically embedded in $X$ is stronger than $U$ being hyperbolic. It means that for some constant $c>0$, we have $d_{K, U} \geq c d_{X \mid U}$, where $d_{X}$ is a given distance on $X$.

In the case where $n=2$, Zaidenberg deduces from this :

THEOREM 3.13. There exist smooth curves of any degree $d \geq 5$ which have hyperbolic, and even hyperbolically embedded complements.

The proof shows that there is an open neighborhood for the analytic topology, in the set of all degree $d$ curves, of the point parameterizing an union of $d \geq 5$ lines in general position, which consists of curves with hyperbolically embedded complement. It uses Green's Theorem above, together with Theorem 1.22, and a careful analysis of what happens to a sequence of holomorphic maps $f_{n}: \mathbb{C} \rightarrow U_{n}:=\mathbb{P}^{2} \backslash C_{n}$ when $C_{n}$ tends to a generic union of $d \geq 5$ lines. This last curve $C_{0}$ is of course not Brody hyperbolic, but the point is that $C_{0} \backslash \operatorname{Sing} C_{0}$ is Brody hyperbolic and that $C_{0}$ has ordinary singularities.

In a completely different spirit, Ciliberto and Zaidenberg constructed recently singular hypersurfaces in $\mathbb{P}^{4}$, of degree $\geq 125$, which are Brody hyperbolic, thus reproving the existence of smooth hyperbolic hypersurfaces in these ranges of degree. The idea is very simple : consider a self-product $C^{3}$, where $C$ is a smooth curve of genus $g \geq 2$. It is clearly hyperbolic. Furthermore it can be sent to a hypersurface in $\mathbb{P}^{4}$ using an adequate linear system. The image $Y$ of this map $\phi: C^{3} \rightarrow \mathbb{P}^{4}$ will not be isomorphic to $C^{3}$, since there is a surface $\Sigma \subset C^{3}$ on which $\phi$ is $2-1$, and a curve $D$ on which $\phi$ is $3-1$. However a holomorphic map from $\mathbb{C}$ to $Y$ would lift to $C^{3}$ if it does not have image in $\Sigma^{\prime}:=\phi(\Sigma)$. So any such map has to take value in $\Sigma^{\prime}$. On then shows that the normalization $\widetilde{\Sigma^{\prime}}$ of $\Sigma^{\prime}$ has high irregularity, so that Bloch's theorem 1.18 allows to show that any entire curve in $\Sigma^{\prime}$ has to be algebraically degenerate. It remains to see that $\Sigma^{\prime}$ cannot contain a rational or elliptic curve. However, using the fact that the triple curve $\phi(D)$ has genus 
$>1$, this would produce a hyperelliptic curve or a double cover of an elliptic curve in $C^{3}$, hence a rational curve or an elliptic curve in the symmetric product $C^{(2)}$, which one can easily show not to exist if $C$ is generic and the genus of $C$ is large enough.

In the case of surfaces, Fujimoto [28] and independently Duval [25] constructed examples of singular surfaces in $\mathbb{P}^{3}$, of degree 8 , which are Brody hyperbolic. Thus by Theorem 1.21, there are smooth surfaces, close to the Fujimoto-Duval, which are hyperbolic. It is still not known whether this is true for generic or general surfaces of degree 8. These surfaces admit a fibration over a curve of genus $\geq 2$, with fiber curves of genus $\geq 2$. The fibration is obtained as the Stein factorization of a linear projection to $\mathbb{P}^{1}$. Their equations is of the form

$$
F^{2}\left(X_{0}, X_{1}, X_{2}\right)+G\left(X_{2}, X_{3}\right)=0,
$$

for generic polynomials $F$ and $G$ of degree 4, resp. 8, in 3, resp. 2 variables. The fiber of the projection $p$ to $\mathbb{P}^{1}$ given by the coordinates $X_{2}, X_{3}$ has for equation $F^{2}\left(X_{0}, X_{1}, X_{2}\right)+\alpha X_{2}^{2}=0$, and so it is the union of two plane quartic curves with equations

$$
F\left(X_{0}, X_{1}, X_{2}\right) \pm \sqrt{\alpha} X_{2}=0 .
$$

The coefficient $\alpha$ vanishes at 8 distinct points of $\mathbb{P}^{1}$, so that the double cover $C$ of $\mathbb{P}^{1}$ ramified along these 8 points is a smooth curve of genus $>1$. As one can see, the Stein factorization of $p$ is in fact well defined, and the fibers all have geometric genus $>1$.

Recently, Shiffman and Zaidenberg [60] have constructed other examples of octic surfaces which are Kobayashi hyperbolic. They are obtained as small deformations of reducible octic surfaces. Note that it is expected that similar results hold for quintic surfaces (Conjecture $1.53)$.

We turn now to other recent results on Kobayashi's conjecture 0.7. Concerning the case of surfaces, it has been solved positively by Demailly and Elgoul [22], and independently by McQuillan [51], although their result are not optimal, as shown by Fujimoto-Duval's example :

TheOREM 3.14. Let $S$ be a general surface in $\mathbb{P}^{3}$ of degree $\geq 21$. Then $S$ is Kobayashi hyperbolic.

The result has been proved by McQuillan for degree $d \geq 36$.

An important idea of the proof is the use of a notion of jet differential, which is slightly different from the Green-Griffiths notion (cf section 1.2.3) and was introduced by Demailly in [21] : the space of $k$-jets $X_{k}$ considered is an explicitly described compactification of the space of smooth unparameterized jets, which can be alternatively defined as 
the space of curvilinear subschemes of lenght $k+1$ of $X$ supported at one point :

$$
X_{k} \supset X_{k}^{\text {curv }}:=\left\{z \subset X, z \cong \operatorname{Spec} \mathbb{C}[t] /\left(t^{k+1}\right)\right\} .
$$

This $X_{k}$ can be constructed as the "Proj" of the sheaf of jet differentials considered by Demailly, which are the jet differentials invariant up to a multiplicative coefficient under the full reparameterization group Aut Spec $\mathbb{C}[t] /\left(t^{k+1}\right)$. As a projective bundle, $X_{k}$ has a line bundle $\mathcal{O}_{X_{k}}(1)$. By definition of $X_{k}$, Demailly's degree $m k$-jet differentials are sections of $\mathcal{O}_{X_{k}}(m)$.

A non constant holomorphic map $f: C \rightarrow X$ from any smooth curve to $X$ admits a lifting $f_{k}: C \rightarrow X_{k}$, which to a point $c$ where $f$ is of maximal rank, associates the restriction of $f$ to the infinitesimal neighborhood of order $k$ of $c$ in $C$.

Green-Griffiths Theorem 1.36 provides in this case :

LEMMA 3.15. Let $L$ be an ample line bundle on $X$ and let $\sigma$ be a section of $\mathcal{O}_{X_{k}}(m) \otimes \pi^{*} L^{-1}$, where $\pi: X_{k} \rightarrow X$ is the structural map. Then for any non constant holomorphic map $f: \mathbb{C} \rightarrow X, \sigma$ vanishes on $\operatorname{Im} f_{k}$.

Consider now the case where $S$ is a surface of general type. RiemannRoch estimates for the sheaves $\pi_{*} \mathcal{O}_{X_{k}}(m)(-L)$ show the following :

Proposition 3.16. (Demailly [21]) If $c_{1}^{2}(S)>\frac{9}{13} c_{2}(S)$, then there is a non zero section of $\pi_{*} \mathcal{O}_{X_{2}}(m)(-L)$, for $m$ large enough.

The assumption on the Chern classes are satisfied by surfaces of degree $\geq 15$ in $\mathbb{P}^{3}$.

The jet space $X_{2}$ is a bundle over $S$, of relative dimension 2. The rest of the proof consists in proving that, if $S$ satisfies Pic $S=\mathbb{Z}$, which is true for general $S$ of degree $\geq 4$ in $\mathbb{P}^{3}$ (the Noether-Lefschetz theorem, cf [69], 3.3.2), and a number of vanishing assumptions, satisfied by a general $S$ of degree $\geq 21$ in $\mathbb{P}^{3}$, the base-locus $B$ of the space of sections of $\pi_{*} \mathcal{O}_{X_{2}}(m)\left(-K_{S}\right)$ has at most 2-dimensional components dominating $S$. Here the assumption on the Picard group is used to describe the possible classes of divisor components in this base-locus.

Having this, the projection $B \rightarrow S$ provides then a multifoliation on $S$, and by Lemma 3.15, entire curves in $S$ must be contained in leaves of this multifoliation. One then applies McQuillan's Theorem 2.9 to conclude that they are algebraically degenerate.

To conclude, it suffices to show that there are no elliptic or rational curves on $S$ for general $S$, but this is known to be true once $S$ is general of degree $\geq 5$ by $[\mathbf{7 2}]$. 
To conclude this section, we conclude with the following beautiful generalization of the Demailly-Elgoul Theorem :

TheOREM 3.17. (Siu, [61]) For any $n>0$, there exists a constant $\delta_{n}$ such that a generic hypersurface $X$ of degree $d \geq \delta_{n}$ in $\mathbb{P}^{n}$ is Kobayashi hyperbolic.

Note the difference between Siu's and Demailly-Elgoul's statement, which lies in the fact that Siu proves his result for generic, i.e. away from a Zariski closed proper subset in the parameter space, while Demailly-Elgoul's Theorem is only for general surfaces, that is, away from a countable union of proper closed algebraic subsets. Indeed, they have first to impose the condition that the Picard group is $\mathbb{Z}$, which holds in the complement of a countable union of proper algebraic subsets, and they also use Xu's result, which is proved only for general surfaces.

The proof uses (Green-Griffiths) jet differentials of order $n-1$ on $X$. The first step is :

Proposition 3.18. Let $q>0$ be fixed. Then for d large enough, there exist an integer $m$ and a non-zero jet differential of order $n-1$ and degree $m$ twisted by $\mathcal{O}_{X}(-q)$ on $X$.

Using the notation $\pi: P_{n-1} \rightarrow X$ for the space of $n-1$-jets, (cf section 1.2.3), this means a section of $\mathcal{O}_{P_{n-1}}(m) \otimes \pi^{*} \mathcal{O}_{X}(-q)$.

Using Theorem 1.36, Theorem 3.17 is reduced to showing that the sections above have an empty base-locus on $P_{n-1}$. This is proved by Siu using the following proposition 3.19 : introduce as in the previous section the universal family $\phi: \mathcal{X} \rightarrow U$ of smooth hypersurfaces of degree $d$ in $\mathbb{P}^{n}$. There is correspondingly a universal family of jet spaces

$$
\phi_{n-1}: \mathcal{P}_{n-1} \rightarrow U, \pi: \mathcal{P}_{n-1} \rightarrow \mathcal{X}
$$

Siu proves next the following:

Proposition 3.19. There exist $c, c^{\prime}$ depending on $n$ but not on $d$, such that the bundle

$$
\left(T_{\mathcal{P}_{n-1}} \otimes \mathcal{O}_{\mathcal{P}_{n-1}}(c) \otimes \pi^{*} \mathcal{O}_{\mathcal{X}}\left(c^{\prime}\right)\right)_{\mid P_{n-1}}
$$

is generated by global sections.

Here $P_{n-1} \subset \mathcal{P}_{n-1}$ is the fiber of $\phi_{n-1}$ over the point $x \in U$ parameterizing $X$. Note that the correct version of this statement should in fact take into account the (mild) singularities of this space (see [62]).

Having this, we let Lie derivative with respect to these vector fields act on the non zero section given by Proposition 3.18, which may be 
chosen to vary holomorphically with $X$. Taking $q$ very large with respect to $c, c^{\prime}$, and using a precise estimate on the number $m$ in Proposition 3.18 , Siu finally gets, by iterating enough this action, a non zero section at any point.

3.2. Calabi-Yau hypersurfaces . We consider now the case of Calabi-Yau hypersurfaces. They are far from being algebraically hyperbolic. On the other hand, it is not known if their Kobayashi pseudodistance or pseudo-volume form are 0, according to conjecture 0.1. We describe results and conjectures by Clemens, and we also explain a method to study sweeping-out of general hypersurfaces by varieties of a given type. This leads to a statement which seems to indicate that Lang's conjecture 2.13 might be wrong.

3.2.1. Rational curves on Calabi-Yau hypersurfaces. We study here rational curves in Calabi-Yau hypersurfaces, that is hypersurfaces of degree $d=n+2$ in $\mathbb{P}^{n+1}$. In contrast with Conjecture 3.9, which concerned hypersurfaces with ample canonical bundle, we have the following result (see $[\mathbf{1 3}])$ :

TheOREM 3.20. (Clemens) A general Calabi-Yau hypersurface of dimension $\geq 2$ contains rational curves of arbitrarily large degree.

We will focus on the dimension 3 case. The same technic applies in higher dimension. Note that for a smooth rational curve $C \subset X$, where $X$ is a Calabi-Yau threefold, the adjunction formula gives deg $N_{C / X}=-2$ and the Riemann-Roch formula then shows that $\chi\left(N_{C / X}\right)=0$. Hence one can expect that the normal bundle of such curve will generically satisfy the conditions

$$
h^{0}\left(N_{C / X}\right)=h^{1}\left(N_{C / X}\right)=0,
$$

which implies that, identifying the curve with $\mathbb{P}^{1}$, the normal bundle has the following shape:

$$
N_{C / X} \cong \mathcal{O}_{\mathbb{P}^{1}}(-1) \oplus \mathcal{O}_{\mathbb{P}^{1}}(-1) .
$$

Such curve will be said to be infinitesimally rigid because it has no infinitesimal deformations in $X$. Theorem 3.20 can then be made more precise :

TheOrem 3.21. A general quintic threefold $X \subset \mathbb{P}^{4}$ contains infinitely many infinitesimally rigid rational curves.

Proof. The idea is to construct explicitly a singular (nodal) quintic, containing infinitely many rigid rational curves not passing through the nodes. These curves are stable under deformations (cf [45]), which 
implies that the relative Hilbert scheme parameterizing such a pair $(C, X), C \subset X$, will be smooth (in fact étale) over the deformation space $U$ of $X$, hence in particular dominating, since both spaces are quasi-projective. The rigidity condition being Zariski open, it will then suffice to consider the complement of the union of those proper algebraic subsets of $U$ which are the images of the closed proper algebraic subsets of the relative Hilbert schemes above where the curve is not anymore infinitesimally rigid, to get the result.

The construction is the following : the singular quintic threefold will contain a plane $P$, which forces generically 16 nodes which are on $P$, and no other singularities. We ask also that a hyperplane section $\Sigma \subset X$ containing $P$ is the union

$$
P \cup S,
$$

where the quartic surface satisfies the following property :

$S$ contains three lines $l_{0}, l_{1}, l_{\infty}$, not meeting Sing $X$ and mutually non-intersecting. Projecting $S$ from $l_{\infty}$ makes it into an elliptic fibration. The line $l_{0}$ will then play the role of the 0 -section, and we can apply then the multiplication map $\cdot m$ by any integer $m$ to $l_{1}$ to get rational curves $C_{m} \subset S$.

It is easy to compute the degree of these curves so as to prove that this degree tends to $\infty$ with $m$. Also, it is clear that only finitely many such curves will pass through one of the nodes of $X$, given a generic $X$ containing $\Sigma$.

It remains to see that for such a generic choice of $X$, infinitely many curves $C_{m}$ are infinitesimally rigid in $X$. This is done as follows : the $K 3$ surface $S$ has trivial canonical bundle, hence the rational curves $C_{m}$ have normal bundle $\mathcal{O}_{\mathbb{P}^{1}}(-2)$ in $S$. The normal bundle of $S$ in $X$ is trivial away from the nodes of $X$, so that there is an extension class $e \in H^{1}\left(S, T_{S}\right) \cong H^{1}\left(S, \Omega_{S}\right)$ associated to the normal bundle sequence :

$$
0 \rightarrow T_{S} \rightarrow T_{X \mid S} \rightarrow \mathcal{O}_{S} \rightarrow 0 .
$$

(Here, to take into account the nodes of $X$, we can either restrict everything to $S \backslash\{$ nodes of $X\}$, or work with the small resolution of $X$ which contains a copy of $S$.) The image $e_{m}$ of $e$ in each group

$$
H^{1}\left(N_{C_{m} / S}\right) \cong H^{1}\left(\Omega_{C_{m}}\right),
$$

via the restriction map $H^{1}\left(\Omega_{S}\right) \rightarrow H^{1}\left(\Omega_{C_{m}}\right)$, is the extension class of the normal bundle sequence

$$
0 \rightarrow N_{C_{m} / S} \rightarrow N_{C_{m} / X} \rightarrow \mathcal{O}_{C_{m}} \rightarrow 0,
$$

and this extension class will be non trivial if and only if the normal bundle of $C_{m}$ in $X$ is isomorphic to $\mathcal{O}_{\mathbb{P}^{1}}(-1) \oplus \mathcal{O}_{\mathbb{P}^{1}}(-1)$. Now, it is 
easy to show that for a generic choice of $X$ containing $\Sigma$, the class $e_{1}$ is non trivial, and computing the cohomology classes of the curves $C_{m}$, it will follow that for infinitely many of the curves $C_{m}$, the class $e_{m}$ is also non trivial.

Clemens (see [17], Lecture 22) conjectured the following :

CONJECTURE 3.22. If $X$ is a general quintic threefold, there are at most finitely many rational curves of given degree in $X$.

This conjecture has been proved up to degree 9 (see [37]). More generally, one might conjecture that in a general Calabi-Yau hypersurface of dimension $\geq 3$, rational curves cover a countable union of Zariski closed proper algebraic subsets of codimension $\geq 2$.

This is supported by the following argument from deformation theory : if $C \subset X$ is a smooth curve, with normal bundle $N_{C / X}$, the obstructions to deform $C$ with $X$ lie in $H^{1}\left(C, N_{C / X}\right)$. Now assume $X$ has trivial canonical bundle and $C$ is rational. Then $h^{1}\left(N_{C / X}\right)=$ $0 \Rightarrow h^{0}\left(N_{C / X}\right)=n-3$, as show adjunction formula and RiemannRoch theorem. So Clemens conjecture is supported by the idea that if there are no obstructions to deform a rational curve $C$ with $X$, then $h^{1}\left(N_{C / X}\right)=0$. The exception $n=2$ is well understood from this point of view. Indeed, curves in a quartic surfaces are obstrued, but the point is that the deformations of the quartic surface in $\mathbb{P}^{3}$ do not provide all the deformations of its complex structure but only an hypersurface in them.

REMARK 3.23. For special quintic, there are interesting one-dimensional families of rational curves, eg one-dimensional families of lines which are not the base of a cone in $X$. (cf $[\mathbf{1}])$.

REMARK 3.24. The conjecture becomes wrong if instead of a quintic threefold, we consider double covers of $\mathbb{P}^{3}$ ramified along an octic surface $S$ (cf Example 2.17). Indeed, consider lines $l$ in $\mathbb{P}^{3}$ which meet $S$ along a 0 -cycle of the form $2 x+2 y+4 z$. It is easy to see that there is a one-dimensional family of such lines, and the normalization of their inverse image in the double cover is rational. Note that these double covers have Picard rank 1, as the quintic threefold does. The interest of this example lies in the fact that these one-parameter families of rational curves survive all deformations of the complex structure, in spite of the deformation argument sketched above.

The following generalizes the above remark, and shows that proving Clemens' Conjecture 3.22 or disproving Lang's Conjecture 2.13 might 
be very hard. Let $X$ be a $K$-trivial complex threefold, and assume that $X$ contains a smooth rational curve $\Delta \cong \mathbb{P}^{1}$ with normal bundle

$$
N_{\Delta / X} \cong \mathcal{O}_{\mathbb{P}^{1}}(-1) \oplus \mathcal{O}_{\mathbb{P}^{1}}(-1) .
$$

Proposition 3.25. Assume there exists a rational curve $\Delta^{\prime} \subset X$, and a neighborhood $U$ of $\Delta \cup \Delta^{\prime}$ in $X$, together with an involution

$$
i: U \rightarrow U
$$

satisfying the following properties :

i) The fixed locus of $i$ is a (smooth) hypersurface of $U$, meeting transversally $\Delta$.

ii) We have $i(\Delta)=\Delta^{\prime} \neq \Delta$.

Then, if the involution $i$ has at least one fixed point on $\Delta, X$ contains a one-parameter family of rational curves.

If the involution $i$ has at least two fixed points $o, o^{\prime}$ on $\Delta$, and the tangent directions to $\Delta^{\prime}$ at $o$ and $o^{\prime}$ are distinct directions in $N_{\Delta / X}$, then $X$ is swept-out by a two-parameter family of elliptic curves.

Note that in the second statement, comparing the projections of the tangent directions to $\Delta^{\prime}$ at $o$ and $o^{\prime}$ in $N_{\Delta / X, o}$ and $N_{\Delta / X, o^{\prime}}$ respectively, makes sense because the vector bundle $N_{\Delta / X}$ is projectively trivial.

Proof. We give the proof under the simplifying assumption that $\Delta$ and $\Delta^{\prime}$ meet only at fixed points of $i$. Let $U^{\prime}$ be the quotient of $U$ under the involution $i$. There is a natural map

$$
\phi: \Delta \rightarrow U^{\prime}
$$

which is the composite of the projection $U \rightarrow U^{\prime}$ and of the immersion of $\Delta$ in $U$. $U^{\prime}$ is smooth along $\operatorname{Im} \phi$. Since $\Delta$ is not tangent to the fixed locus of $i, \phi$ is also an immersion. The normal bundle $N^{\prime}$ of $\phi$ fits into an exact sequence

$$
0 \rightarrow N_{\Delta / X} \rightarrow N^{\prime} \rightarrow \oplus_{x \in Z} \mathbb{C}_{x} \rightarrow 0,
$$

where $Z$ is the set of fixed points of $i$ on $\Delta$, and the kernel of the induced map

$$
N_{\Delta / X} \otimes \mathcal{O}_{x} \rightarrow N^{\prime} \otimes \mathcal{O}_{x}
$$

is generated by the image of $T_{\Delta^{\prime}, x}=i_{*}\left(T_{\Delta, x}\right.$ in $N_{\Delta / X} \otimes \mathcal{O}_{x}$. It follows immediately from (3.24) that $H^{1}\left(N^{\prime}\right)=0$ and that $h^{0}\left(N^{\prime}\right) \geq m$, where $m$ is the number of fixed points of $i$ which lie on $\Delta$.

Note that the image $I$ in $U^{\prime}$ of the fixed locus of $i$ is a smooth hypersurface, and that $\phi(C)$ is tangent to first order to $I$ at each $\phi(x)$, $x \in Z$. 
Assume first that $Z$ contains at least one point $z$. Let $z_{1}, \ldots, z_{m-1}$ be the remaining points of $Z$. Consider the subspace $M$ of the space of deformations of $\phi$ consisting of those $\phi_{t}: \mathbb{P}^{1} \rightarrow U^{\prime}$ which remain tangent to $I$ at $z_{i}, 1 \leq i \leq m-1$. This space is of codimension $\leq m-1$ in the space of deformations of $\phi$, which is smooth of dimension $m+3$. Hence $\operatorname{dim} M \geq 4$. On the other hand, a point $\phi_{t} \in M$ provides a rational curve

$$
C_{t} \subset U,
$$

obtained as follows : since for $\phi_{t}$ close enough to $\phi, \phi_{t}\left(\mathbb{P}^{1}\right)$ has simple tangency to $I$ at $z_{i}, 1 \leq i \leq m-1$, there are at most two transverse intersection points in $\phi_{t}\left(\mathbb{P}^{1}\right) \cap I$, hence the normalization of the double cover of $\mathbb{P}^{1}$ ramified along $\phi_{t}^{-1}(I)$ is a rational curve, which sends to $U$. Thus we have constructed a four dimensional family of maps from $\mathbb{P}^{1}$ to $U$, or a one dimensional family of rational curves in $U$. These cuves are deformations of the curve $\Delta \cup_{x} \Delta^{\prime}$.

We consider now the second case. Introduce the bundle $N^{\prime}$ which is the elementary transform of $N_{\Delta / X}$ at $O$ and $o^{\prime}$, fitting in the exact sequence :

$$
0 \rightarrow N_{\Delta / X} \rightarrow N^{\prime} \rightarrow \mathbb{C}_{o} \oplus \mathbb{C}_{o^{\prime}} \rightarrow 0,
$$

where the kernels of the induced maps

$$
N_{\Delta / X} \otimes \mathcal{O}_{o} \rightarrow N^{\prime} \otimes \mathcal{O}_{o}, N_{\Delta / X} \otimes \mathcal{O}_{o^{\prime}} \rightarrow N^{\prime} \otimes \mathcal{O}_{o^{\prime}}
$$

are given by the projections of the tangent direction of $\Delta^{\prime}$ at the considered point. By our assumption, the bundle $N^{\prime}$ is trivial. On the other hand, let us consider the space of deformations $\phi_{t}$ of $\phi$ which have the property that $\phi_{t}\left(\mathbb{P}^{1}\right)$ remains tangent to $I$ at the remaining points $z_{i} \in Z \backslash\left\{o, o^{\prime}\right\}$. Modulo Aut $\mathbb{P}^{1}$, this space has tangent space $h^{0}\left(N^{\prime}\right)$ and obstruction space $h^{1}\left(N^{\prime}\right)$. Hence it has dimension 2, and furthermore, the fact that $N^{\prime}$ is trivial implies that the maps $\phi_{t}$ for $t$ in this space sweep-out a neighborhood of $\phi(\Delta)$. On the other hand, for such a $\phi_{t}$, there are at most two transverse intersection points in $\phi_{t}\left(\mathbb{P}^{1}\right) \cap I$, hence the double cover of $\mathbb{P}^{1}$ ramified along $\phi_{t}^{-1}(I)$ has for normalization an elliptic curve which sends to $U$ over $\operatorname{Im} \phi_{t}$. Hence we have a two-dimensional family of elliptic curves which deform the singular reducible elliptic curve $\Delta \cup_{o, o^{\prime}} \Delta^{\prime}$ in $U$ and sweep out $U$.

3.2.2. Sweeping out a general hypersurface by subvarieties. We shall now describe an argument which allows to study rational maps from varieties which are members of a given family to general hypersurfaces. More precisely, let us introduce the following definition : 
DeFinition 3.26. Let $S$ be a quasi-projective variety and $f: \mathcal{Y} \rightarrow S$ be a smooth projective map of fibre dimension $r$. We say that a variety $X$ is rationally swept-out by varieties of the family $\mathcal{Y}$ if there exists a $n-r$-dimensional quasi-projective variety $B$, a map $\rho: B \rightarrow S$, and a dominating rational map :

$$
\phi: \mathcal{Y}_{B} \rightarrow X \text {. }
$$

Here $\mathcal{Y}_{B}:=\mathcal{Y} \times{ }_{S} B$. This $\phi$ will then be generically finite since both sides have the same dimension. In particular it is generically finite on the generic fibre $Y_{b}$ of the family $f: \mathcal{Y}_{B} \rightarrow B$. Our goal is to study rational sweeping out of general hypersurfaces of degree $d$ and dimension $n$ by varieties parameterized by a basis of small dimension. Our result [68] is the following:

TheOREM 3.27. Fix an integer $1 \leq r \leq d$. Let $\gamma=\frac{r-1}{2}, r$ odd, or $\gamma=\frac{r}{2}, r$ even, that is $\gamma$ is the round-up of $\frac{r-1}{2}$. Let $\mathcal{Y} \rightarrow \mathcal{S}$, $\operatorname{dim} \mathcal{S}=C$, be a family of $r$-dimensional smooth projective varieties. Then the general hypersurface of degree $d$ in $\mathbb{P}^{n+1}$ is not rationally swept out by varieties parameterized by $\mathcal{S}$ if

$$
(d+1) r \geq 2 n+C+2,(\gamma+1) d \geq 2 n-r+1+C .
$$

Let us sketch the method to prove this : to start with, observe that by an easy countability argument involving the countably many Chow varieties parameterizing an hypersurface $X$, a point $s$ of $S$, and the graph of a generically finite rational map

$$
\phi: Y_{s} \rightarrow X,
$$

it suffices to show that under the assumptions of Theorem 3.27, there do not exist a quasi-projective variety $\mathcal{B}$, a map $\rho: \mathcal{B} \rightarrow S$, a morphism $m: \mathcal{B} \rightarrow U$ and a dominating rational map

$$
\Phi: \mathcal{Y}_{\mathcal{B}} \rightarrow \mathcal{X}
$$

which is over $U$, i.e such that $\pi \circ \Phi=m \circ f$. Here the notations are as before : $U$ parameterizes smooth hypersurfaces of degree $d, \pi: \mathcal{X} \rightarrow U$ is the universal hypersurface, and $f: \mathcal{Y}_{\mathcal{B}} \rightarrow \mathcal{B}$ is the pull-back of the family $\mathcal{Y}$ via the morphism $\rho$.

So let us assume by contradiction that such data are given. Observe that for $s \in S$ fixed, the fibre $\rho^{-1}(s)=: \mathcal{B}_{s}$ has codimension $\leq C$ in $\mathcal{B}$. We may assume shrinking $\mathcal{B}$ if necessary that $\mathcal{B}, \mathcal{B}_{s}$ are smooth and that $m$ is of maximal rank along $\mathcal{B}_{s}$. Then the corank of $m_{s}=m_{\mid \mathcal{B}_{s}}: \mathcal{B}_{s} \rightarrow U$ is $\leq C$, and we may assume that it is constant. 
Next, restrict $\Phi$ to $\mathcal{Y}_{s}:=f^{-1}\left(\mathcal{B}_{s}\right)$ and denote it by $\Phi_{s}$. Note that $\mathcal{Y}_{s} \cong Y_{s} \times \mathcal{B}_{s}$. Hence we get a rational map

$$
\Phi_{s}: Y_{s} \times \mathcal{B}_{s} \rightarrow \mathcal{X}_{s}, \mathcal{X}_{s}:=\mathcal{X} \times_{U} \mathcal{B}_{s},
$$

which is over $\mathcal{B}_{s}$. This means that its graph $\Gamma_{\Phi_{s}}$ lies in

$$
Y_{s} \times \mathcal{B}_{s} \times_{\mathcal{B}_{s}} \mathcal{X}_{s}
$$

that is in

$$
Y_{s} \times \mathcal{X}_{s}
$$

Note that $\Gamma_{\Phi_{s}}$ has codimension $n$ in the above variety. Hence it has a cohomology class $\gamma_{s}$ in $H^{2 n}\left(Y_{s} \times \mathcal{X}_{s}, \mathbb{Q}\right)$.

We shall be mainly interested in the Künneth component $\gamma_{s, r}$ of $\gamma_{s}$ in $H^{r}\left(Y_{s}, \mathbb{Q}\right) \otimes H^{2 n-r}\left(\mathcal{X}_{s}, \mathbb{Q}\right)$. Now we have the following theorem, which is an easy refinement of Nori's connectivity theorem [56], or more precisely of the explicit Nori theorem for hypersurfaces, which can be found eg in [69], 8.3.2. Let $m: \mathcal{M} \rightarrow U$ be a morphism, where $\mathcal{M}$ is smooth quasi-projective. We assume that corank $m$ is constant equal to $C$. Let

$$
\mathcal{X}_{\mathcal{M}}:=\mathcal{X}_{U} \times_{U} \mathcal{M}
$$

and let

$$
j: \mathcal{X}_{\mathcal{M}} \hookrightarrow \mathcal{M} \times \mathbb{P}^{n+1}
$$

be the natural embedding.

THEOREM 3.28. i) Assume that

$$
(d+1) r \geq 2 n+C+2 .
$$

Then, the restriction map

$$
j^{*}: F^{n} H^{2 n-r}\left(\mathcal{M} \times \mathbb{P}^{n+1}, \mathbb{C}\right) \rightarrow F^{n} H^{2 n-r}\left(\mathcal{X}_{\mathcal{M}}, \mathbb{C}\right)
$$

is surjective.

ii) If

$$
(\gamma+1) d \geq 2 n+1-r+C,
$$

then for any $i \geq 1$, the restriction map

$$
j^{*}: H^{2 n-r-i}\left(\mathcal{M} \times \mathbb{P}^{n+1}, \mathbb{C}\right) \rightarrow H^{2 n-r-i}\left(\mathcal{X}_{\mathcal{M}}, \mathbb{C}\right)
$$

is surjective.

Here the Hodge filtration $F$ is the Hodge filtration defined on the cohomology of a smooth quasi-projective variety [20].

From this theorem, we deduce :

Corollary 3.29. The class $\gamma_{s, r}$ vanishes in

$$
H^{r}\left(Y_{s}, \mathbb{Q}\right)_{t r} \otimes\left(H^{2 n-r}\left(\mathcal{X}_{s}, \mathbb{Q}\right) / H^{2 n-r}\left(\mathbb{P}^{n+1} \times \mathcal{B}_{s}, \mathbb{Q}\right)\right) \text {. }
$$


Here the suffix "tr" denotes the transcendental part of the cohomology group $H^{r}\left(Y_{s}, \mathbb{Q}\right)$, that is the quotient of $H^{r}\left(Y_{s}, \mathbb{Q}\right)$ by all the sub-Hodge structures which are not of Hodge level $r$, that is which have no $(r, 0)$-part.

Proof of Corollary. Indeed, $\gamma_{s, r}$ is a Hodge class. This means that, viewed by Poincaré duality as an element of

$$
\operatorname{Hom}\left(H^{r}\left(Y_{s}, \mathbb{Q}\right), H^{2 n-r}\left(\mathcal{X}_{s}, \mathbb{Q}\right)\right)
$$

it will be in the subspace

$$
\operatorname{Hom}_{H S}\left(H^{r}\left(Y_{s}, \mathbb{Q}\right), W_{0} H^{2 n-r}\left(\mathcal{X}_{s}, \mathbb{Q}\right)\right),
$$

consisting of morphisms of Hodge structures (of bidegree $(n-r, n-r)$ ) from $H^{r}\left(Y_{s}, \mathbb{Q}\right)$ to the subspace $W_{0} H^{2 n-r}\left(\mathcal{X}_{s}, \mathbb{Q}\right)$, which is the pure part (of Hodge weight $2 n-r$ ) of $H^{2 n-r}\left(\mathcal{X}_{s}, \mathbb{Q}\right.$ ). Now, we can apply Theorem 3.28, i) to $m_{s}: \mathcal{B}_{s} \rightarrow U$, since we know that the corank of $m_{s}$ is $\leq C$. This says that $F^{n}\left(H^{2 n-r}\left(\mathcal{X}_{s}, \mathbb{C}\right) / H^{2 n-r}\left(\mathbb{P}^{n+1} \times \mathcal{B}_{s}, \mathbb{C}\right)\right)=0$, and this implies that the Hodge structure on

$$
W_{0} H^{2 n-r}\left(\mathcal{X}_{s}, \mathbb{Q}\right) / H^{2 n-r}\left(\mathbb{P}^{n+1} \times \mathcal{B}_{s}, \mathbb{Q}\right)
$$

has no $H^{p, q}$-part for $p \geq n$. It follows that any morphism of Hodge structures of bidegree $(n-r, n-r)$ from $H^{r}\left(Y_{s}, \mathbb{Q}\right)$ to $W_{0} H^{2 n-r}\left(\mathcal{X}_{s}, \mathbb{Q}\right) / H^{2 n-r}\left(\mathbb{P}^{n+1} \times\right.$ $\left.\mathcal{B}_{s}, \mathbb{Q}\right)$ vanishes on $H^{r, 0}\left(Y_{s}\right)$, and thus on $H^{r}\left(Y_{s}\right)_{t r}$.

The conclusion of the proof of Theorem 3.27 is now roughly the following: the rational map $\Phi$ of (3.28) has a graph

$$
\Gamma_{\Phi} \subset \mathcal{Y}_{\mathcal{B}} \times_{U} \mathcal{X}
$$

and has codimension $n$ here. Its class $\left[\Gamma_{\Phi}\right] \in H^{2 n}\left(\mathcal{Y}_{\mathcal{B}} \times_{U} \mathcal{X}, \mathbb{Q}\right)$ restricts on $\mathcal{Y}_{s} \times_{\mathcal{B}_{s}} \mathcal{X}_{s}$ to the class $\gamma_{s}$ considered above. On the other hand, let us fix $u \in U$, and let $B_{u}:=m^{-1}(u)$. Then $\Phi$ restricts to the surjective dominating map generically finite on fibers :

$$
\Phi_{u}: \mathcal{Y}_{u} \rightarrow X_{u},
$$

where $\mathcal{Y}_{u}:=\mathcal{Y} \times_{S} B_{u}$. Now $\Phi_{u}$ being dominating, and the variety $X_{u}$ having a non-trivial $H^{n, 0}$ group, $\Phi_{u}$ induces a non-zero (in fact injective) map

$$
\Phi_{u}^{*}: H^{n}\left(X_{u}, \mathbb{Q}\right)_{t r} \rightarrow H^{n}\left(\mathcal{Y}_{u}, \mathbb{Q}\right),
$$

which has, again by a Hodge level argument, a non-zero induced component in

$$
\operatorname{Hom}\left(H^{n}\left(X_{u}, \mathbb{Q}\right)_{t r}, H^{n-r}\left(B_{u}, R^{r} f_{*} \mathbb{Q}_{t r}\right)\right)
$$


This implies that the class $\left[\Gamma_{\Phi}\right]$ is non trivial, even modulo the image of $H^{2 n}\left(\mathcal{Y}_{\mathcal{B}} \times \mathbb{P}^{n+1}, \mathbb{Q}\right)$, in $H^{2 n-r}\left(\mathcal{X}_{\mathcal{B}}, R^{r} f_{*} \mathbb{Q}_{t r}\right)$.

Applying the vanishing statement given in part ii) of Theorem 3.28 and a Leray spectral sequence argument to the map $\rho \circ \pi: \mathcal{X}_{\mathcal{B}} \rightarrow \mathcal{S}$, we conclude then that the restriction $\gamma_{s}$ of $[\Phi]$ also does not vanish in

$$
H^{r}\left(Y_{s}, \mathbb{Q}\right)_{t r} \otimes\left(H^{2 n-r}\left(\mathcal{X}_{s}, \mathbb{Q}\right) / H^{2 n-r}\left(\mathbb{P}^{n+1} \times \mathcal{B}_{s}, \mathbb{Q}\right)\right),
$$

which contradicts Corollary 3.29.

3.2.3. Lang's and Clemens' conjectures contradict . Let us apply Theorem 3.27 to the case where the family $\mathcal{Y} \rightarrow \mathcal{S}$ is a family of polarized abelian varieties. Hence we may assume that $C=\operatorname{dim} \mathcal{S} \leq$ $\frac{r(r+1)}{2}$. We shall be only interested in the case where $d=n+2$, namely the case of $K$-trivial hypersurfaces, since an hypersurface with ample canonical bundle cannot be rationally swept out by abelian varieties. Theorem 3.27 then gives the following :

THEOREM 3.30. A general hypersurface of degree $d=n+2$ in $\mathbb{P}^{n+1}$ is not rationally swept out by abelian varieties of dimension $r \geq 2$.

Indeed, it suffices to check the inequalities

$$
(n+3) r \geq 2 n+\frac{r(r+1)}{2},(\gamma+1)(n+2) \geq 2 n-r+1+\frac{r(r+1)}{2},
$$

for $n \geq r \geq 2$, where $\gamma$ is round-up of $\frac{r-1}{2}$.

Let us now come back to Lang's conjecture 2.13. Note first of all that for $X$ a variety with trivial canonical bundle (or more generally with Kodaira dimension $\geq 0$ ), it can be restated as follows :

Conjecture 3.31. A variety $X$ which is not of general type and has Kodaira dimension $\geq 0$ should be rationally swept out by abelian varieties of dimension $r \geq 1$.

Indeed, since a multiple of the canonical bundle of $X$ is effective, it is easy to see that a rational map $\phi: A \rightarrow X$ whose infinitesimal deformations generate generically the normal bundle, should factor as

$$
A \rightarrow B \stackrel{\psi}{\rightarrow} \rightarrow X
$$

where the first map is a quotient map to an abelian variety $B$ and the second map is generically finite. So if $X$ is covered by rational images of non-constant maps from an abelian variety, it must be covered by rational images of non-constant generically finite maps from an abelian variety. 
Now, Theorem 3.30 says that a general Calabi-Yau hypersurface is not rationally swept out by abelian varieties of dimension $\geq 2$. So we have :

Corollary 3.32. If Lang's conjecture is true, a general CalabiYau hypersurface is swept out by elliptic curves.

Next we have the following (see [68] or [17], lecture 22) :

Lemma 3.33. A general Calabi-Yau hypersurface of dimension $n \geq$ 2 is not rationally swept out by elliptic curves of constant modulus.

Proof. Indeed, there would be otherwise a dominant rational map

$$
\phi: B \times E \rightarrow X,
$$

where $X$ is generic, $E$ is an elliptic curve, and $\operatorname{dim} B=n-1$. This would induce an injective morphism or rational Hodge structure

$$
H^{n}(X, \mathbb{Q})_{\text {prim }} \rightarrow H^{1}(E, \mathbb{Q}) \otimes H^{n-1}(B, \mathbb{Q}),
$$

since the Hodge structure on $H^{n}(X, \mathbb{Q})_{\text {prim }}$ is simple for general $X$.

It is easy to disprove the existence of such a morphism for general $X$ by an infinitesimal argument concerning the variations of Hodge structure on $H^{n}(X, \mathbb{Q})_{\text {prim }}$.

Hence we get the following corollary :

COROLlary 3.34. If Lang's conjecture 2.13 is true, a general CalabiYau hypersurface of dimension $n \geq 2$ has a uniruled divisor.

Proof. (See also [17], lecture 22) Indeed, by Corollary 3.32, such an $X$ should be rationally swept out by elliptic curves, and by Lemma 3.33 , the elliptic curves sweeping out $X$ cannot be of constant modulus. This means that there exist a $n$-dimensional projective variety $E$, a morphism $f: E \rightarrow B$ whose general fiber is an elliptic curve, and a surjective morphism $\phi: E \rightarrow X$. Furthermore we may assume that the classifying map $j: B \rightarrow \mathbb{P}^{1}$ is well-defined and non constant. Now, the divisor $(j \circ f)^{-1}(\infty)$ is uniruled, and since this divisor is algebraically equivalent to $(j \circ f)^{-1}(t)$ for any $t \in \mathbb{P}^{1}$, its image in $X$ must contain a divisor, which is also uniruled.

In conclusion, taking for example $n=3$, we proved that Lang's conjecture 2.13 and Clemens' conjecture 3.22 on the finiteness of rational curves of a given degree, contradict. 


\section{References}

[1] A. Albano, S. Katz. Van Geemen's families of lines on special quintic threefolds, Manuscripta Math. 70 (1991) 183-188.

[2] A. Beauville, R. Donagi. La variété des droites d'une hypersurface cubique de dimension 4, C. R. Acad. sci. Paris, Série 1, 301 (1985), 703-706.

[3] A. Beauville. Variétés dont la première classe de Chern est nulle, Journal of Differential Geometry 18 (1983), 755-782.

[4] F. Bogomolov. Families of curves on a surface of general type, Soviet Math. Dokl. Vol. 18 (1977), No 5.

[5] R. Brody. Compact manifolds and hyperbolicity, Trans. Amer. Math. Soc. 235 (1978), 213-219.

[6] R. Brody, M. Green. A family of smooth hyperbolic surfaces in $\mathbb{P}^{3}$, Duke Math. J. 44 (1977), 873-874.

[7] F. Campana. Connexité rationnelle des variétés de Fano, Annales de l'ENS (1992).

[8] F. Campana. Special varieties, preprint 2001, to appear in Annales de l'Institut Fourier.

[9] S. Cantat. Deux exemples concernant une conjecture de Serge Lang, CR Acad. Sci. Paris Sér. I Math. 330 (2000), no. 7, 581-586

[10] J. Carlson, Ph. Griffiths. Infinitesimal variations of Hodge structures and the global Torelli problem, in Journées de géométrie algébrique, (A. Beauville Ed.), Sijthoff-Nordhoff, (1980), 51-76.

[11] L. Chiantini, A.-F. Lopez, Z. Ran. Subvarieties of generic hypersurfaces in any variety, Math. Proc. Cambr. Phil. Soc. 2002.

[12] C. Ciliberto, M. Zaidenberg. 3-fold symmetric products of curves as hyperbolic hypersurfaces in $\mathbb{P}^{4}$, preprint 2002.

[13] H. Clemens. Homological equivalence, modulo algebraic equivalence, is not finitely generated, Inst. Hautes Etudes Sci. Publ. Math. vol. 58 (1983) pp 1938.

[14] H. Clemens. Curves in generic hypersurfaces, Ann. Sci. École Norm. Sup. 19 (1986), 629-636.

[15] H. Clemens, Ph. Griffiths. The intermediate Jacobian of the cubic threefold, Annals of Math. 95 (1972), 281-356.

[16] H. Clemens, Z. Ran. Twisted genus bounds for subvarieties of generic hypersurfaces, to appear Amer. J. Math.

[17] H. Clemens, J. Kollár, S. Mori. Higher dimensional complex geometry, Astérisque 166, SMF (1988).

[18] L. Clozel, E. Ullmo. Correspondances modulaires et mesures invariantes, To appear in Journal für die reine und angewandte Mathematik.

[19] O. Debarre. On Varieties With Ample Cotangent Bundle, preprint math.AG/0306066.

[20] P. Deligne. Théorie de Hodge II, Publ. Math. IHES 40 (1971), 5-57.

[21] J.-P. Demailly. Algebraic criteria for Kobayashi hyperbolic projective varieties and jet differentials, in Proceedings of Symposia in Pure Mathematics volume 62.2, 1997, 285-360.

[22] J. -P. Demailly, J. El Goul, Hyperbolicity of generic surfaces of high degree in projective 3-space, Amer. J. Math. 122 (2000), 515-546. 
[23] J.-P. Demailly, L. Lempert, B. Shiffman. Algebraic approximations of holomorphic maps from Stein domains to projective manifolds, Duke Math. J., vol. 76, 2, 1994, 332-363.

[24] J. Denef, F. Loeser. Germs of arcs on singular algebraic varieties and motivic integration, Invent. Math. 135 (1999), 201-232.

[25] J. Duval. Letter to J.-P. Demailly, oct. 1999 (unpublished).

[26] L. Ein. Subvarieties of generic complete intersections, Invent. Math. 94 (1988), 163-169.

[27] D. A. Eisenman. Intrinsic measures on complex manifolds and holomorphic mappings, Mem. Amer. Math. Soc. 96 (1970).

[28] H. Fujimoto. A family of hyperbolic hypersurfaces in the complex projective space, in The Chuang special Issue, Complex Variables Theory Appl. 43 (2001), 273-283.

[29] T. Graber, J. Harris, J. Starr. Families of rationally connected varieties, to appear in JAMS.

[30] T. Graber, J. Harris, B. Mazur, J. Starr. Rational connectivity and sections of families over curves, to appear Annales de l'ENS.

[31] M. Green, P. Griffiths. Two applications of algebraic geometry to entire holomorphic mappings, in The Chern symposium 1979, Hsiang, Kobayashi, Singer, Weinstein Eds, Springer-Verlag 1980, 41-74.

[32] M. Green. A new proof of the explicit Noether-Lefschetz theorem, J. Diff. Geom. 27 (1988) 155-159.

[33] M. Green. Holomorphic maps to complex tori, Amer. J. Math. 100,(1978),615620.

[34] M. Green. Holomorphic maps into $\mathbb{P}^{n}$ omitting hyperplanes, Trans. Amer. Math. Soc. 169 (1972), 89-103.

[35] Ph. Griffiths. Holomorphic mappings into canonical algebraic varieties, Ann. of Math. 98 (1971), 439-458.

[36] Ph. Griffiths. Periods of certain rational integrals, I, II, Ann. of Math. 90 (1969) 460-541.

[37] T. Johnsen, S. Kleiman. Rational curves of degree at most 9 on a general quintic threefold, Commun. Algebra 24 (1996) 2721-2753.

[38] J.-P. Jouanolou. Hypersurfaces solutions d'une équation de Pfaff algébrique, Math. Ann. 232 (1978) 239-245.

[39] Y. Kawamata. Abundance theorem for minimal threefolds, Inv. Math. 108, 229-246 (1992).

[40] Y. Kawamata. D-equivalence and $K$-equivalence, preprint math.AG/0205287, to appear J. Diff. Geom.

[41] S. Kobayashi. Hyperbolic manifolds and holomorphic mappings, Marcel Dekker, New-York 1970.

[42] S. Kobayashi. Intrinsic distances, measures and geometric function theory, Bull. Amer. Math. Soc. 82 (1976), 357-416.

[43] S. Kobayashi, T. Ochiai. Mappings into compact complex manifolds with negative first Chern class, J. Math. Soc. Japan 23 (1971), 137-148.

[44] S. Kobayashi, T. Ochiai. Meromorphic mappings into complex manifolds of general type, Invent. Math. 31, 1975,7-16.

[45] K. Kodaira : On stability of compact submanifolds of complex manifolds, Amer. J. of Math. vol. 85 (1963). 
[46] J. Kollár. Rational Curves on Algebraic Varieties, Ergebnisse Der Mathematik Und Ihrer Grenzgebiete 3 Folge Bd 32 (1996).

[47] J. Kollár, Y. Miyaoka, S. Mori. Rationally connected varieties, J. Alg. Geom. 1 (1992), 429-448.

[48] S. Lang. Hyperbolic and diophantine Analysis, Bulletin of the American Math. Soc. Vol. 14, 2, (1986), 159-205.

[49] E. Looijenga. Motivic measures, Séminaire Bourbaki, 52ème année, 1999-2000, $n^{0} 874$.

[50] M. McQuillan. Diophantine approximations and foliations, Publ. IHES 87 (1998), 121-174.

[51] M. McQuillan. Holomorphic curves on hyperplane sections of 3-folds, Geom. Funct. Anal. 9 (1999), 370-392.

[52] Y. Miyaoka. On the Kodaira dimension of minimal threefolds, Math. Annalen 281, (1988) 325-332.

[53] Y. Miyaoka, T. Peternell. Geometry of higher dimensional algebraic varieties, DMV Seminar Band 26, Birkhäuser (1997).

[54] D. Mumford. Rational equivalence of zero-cycles on surfaces, J. Math. Kyoto Univ. 9 (1968), 195-204.

[55] V. V. Nikulin. On rational maps between $K 3$ surfaces, in Constantin Caratheodory: an international tribute, World Scientific Publ. Co. (1991) 964995.

[56] M. Nori. Algebraic cycles and Hodge theoretic connectivity, Invent. Math. 111 (1993) 349-373.

[57] G. Pacienza. Rational curves on general projective hypersurfaces, To appear in Journal of Algebraic Geometry.

[58] H. Royden. Remarks on the Kobayashi metric, in Several complex variables II, Lecture Notes in Math. 185, Springer-Verlag (1971), 125-137.

[59] B. Shiffmann, M. Zaidenberg. Hyperbolic hypersurfaces in $\mathbf{P}_{n}$ of FermatWaring type. Proc. Amer. Math. Soc. 130 (2002), 2031-2035.

[60] B. Shiffmann, M. Zaidenberg. New examples of hyperbolic octic surfaces in $\mathbb{P}^{3}$, preprint 2003, math.AG/0306360.

[61] Y-T. Siu. Some recent transcendental techniques in algebraic and complex geometry, in Proceedings of the 2002 International Congress of Mathematicians, Vol 1 (Beijing), pp.439-448.

[62] Y.-T. Siu, Hyperbolicity of generic high-degree hypersurfaces in complex projective spaces, Preprint 2002.

[63] E. Viehweg, Kang Zuo, On the Brody hyperbolicity of moduli spaces for canonically polarized manifolds, math.AG/0101004.

[64] C. Voisin. Théorème de Torelli pour les cubiques de $\mathbb{P}^{5}$, Invent. Math. 86 (1986), 577-601.

[65] C. Voisin. On a conjecture of Clemens on rational curves on hypersurfaces, Journal of Differential Geometry 44 (1996) 200-214.

[66] C. Voisin. Correction to "On a conjecture of Clemens on rational curves on hypersurfaces", J. Differential Geometry 49 (1998), 601-611.

[67] C. Voisin. $K$-correspondences and intrinsic pseudo-volume forms, to appear in the Proceedings of the Fano conference.

[68] C. Voisin. A geometric application of Nori's connectivity theorem, To appear in Annali della Scuola Norm. Sup. di Pisa. 
[69] C. Voisin. Hodge Theory and Complex Algebraic Geometry II, Cambridge University Press 2003.

[70] C. Voisin. Variations de structure de Hodge et zéro-cycles sur les surfaces générales, Mathematische Annalen 299, 77-103 (1994).

[71] C-L Wang. $K$-equivalence in birational geometry, preprint 2002.

[72] G. Xu. Subvarieties of general hypersurfaces in projective space, J. Differential Geometry 39 (1994) 139-172.

[73] G. Xu. On the complement of a generic curve in the projective plane, Amer. J. of Math. (1996), 611-620.

[74] S.-T. Yau. Intrinsic measures on compact complex manifolds, Math. Ann. 212 (1975), 317-329.

[75] M. Zaidenberg. Stability of hyperbolic imbeddedness and construction of examples, Math. USSR Sbornik, Vol. 63 (1989), No 2.

[76] M. Zaidenberg. Hyperbolicity in projective spaces, International Symp. "Holomorphic Mappings, Diophantine Geometry and related Topics (Kyoto 1992), Surikaisekikenkyusho Kokyuroku 819 (1993), 136-156.

[77] M. Zaidenberg. The complement of a generic hypersurface of degree $2 n$ in $\mathbb{P}^{n}$ is not hyperbolic, Siberian Math. J. 28 (1987), 425-432. 